\title{
The effect of target contrast on the attentional blink
}

\author{
FOOK K. CHUA \\ National University of Singapore, Singapore
}

\begin{abstract}
In this set of five rapid serial visual presentation experiments, observers identified one or two target letters that were embedded in a stream of distractors. Target contrasts were varied, and their effects on the attentional blink $(\mathrm{AB})$ were examined. Target identification improved when its contrast was increased. But whereas an increase in the first target's (T1) contrast facilitated its identification, the recovery of the second target (T2) was paradoxically hampered (Experiments 2 and 5). Similarly, identification of the target suffered when the preceding singleton's contrast was increased (Experiment 1). The $\mathrm{AB}$ was eliminated by inserting a blank after a low-contrast, but not a high-contrast, T1 (Experiment 5). Increasing T2's contrast attenuated the blink (Experiment 3) and compensated the larger $\mathrm{AB}$ caused by a high-contrast T1 (Experiment 4). In all, these results showed that attention continued to be engaged as long as the target's contrast prolonged its perceptibility. When the high-contrast target was $\mathrm{T} 1$, a larger $\mathrm{AB}$ was produced; when it was $\mathrm{T} 2$, there was protection from substitution masking.
\end{abstract}

Experiments in which the rapid serial visual presentation (RSVP) paradigm has been used have revealed interesting limitations in the information-processing system. The paradigm consists of presenting stimuli (say, letters) one at a time, each for a short duration $(\approx 100 \mathrm{msec})$, and all at the same location. The observer is required to identify (or detect) one or several targets embedded in a stream of distractors (say, red letters among black distractors). This brief presentation poses no problem when a single target needs to be recovered. Indeed, multiple targets can all be retrieved successfully as long as their temporal separation (lag) is large $(>600 \mathrm{msec})$. But when the lag is short, successful identification of the first target (T1) compromises the recovery of subsequent targets. These limitations have been shown to be attentional, and the period wherein processing appears to be seriously hampered has been dubbed the attentional blink (AB; Raymond, Shapiro, \& Arnell, 1992).

As a way of motivating the experiments in this report, the $\mathrm{AB}$ issue is framed generally as a problem of passing attentional control effectively from one target to another. In this light, the AB may be seen as attention's failing to shift immediately to a new target just as soon as it appears. Because the target appears only fleetingly, such a delay could hamper its recovery. The question is the following: What limits attention shifting immediately to a new target? As in Posner and Petersen (1990), an attentional shift may be conceptualized as follows. Attention

This research was supported by Grant R-107-000-042-112 from the National University of Singapore. Portions of this work were presented at the 24th European Conference of Visual Perception in Kusadasi, Turkey. Correspondence concerning this article should be addressed to F. K. Chua, Department of Psychology, National University of Singapore, Kent Ridge Crescent, Singapore 119260 (e-mail: fkchua@leonis. nus.edu.sg). must first disengage from its original locus before the shift to the new locus can begin. Once the shift has been executed, attention must reengage at the new locus. (In the single-stream RSVP, in which all objects appear in the same spatial location, the spatial shift may be obviated, but attention still has to disengage from the previous target before it can engage the new one.) Each of these steps takes time to execute, and a delay in any would compromise target recovery. Several recent models, proposed to explain the $\mathrm{AB}$ phenomenon, may be viewed from this Posner and Petersen framework.

One view is that the delay in attentional engagement of the second target (T2) is due to the processing demands imposed by $\mathrm{T} 1$. There are several variations of this view. D. E. Broadbent and M. H. P. Broadbent's (1987) model may be seen as an early variant, and the two-stage model (Chun \& Potter, 1995), which fleshes out many of the basic ideas more explicitly, as a more recent one. The two-stage model assumes that each item in the RSVP stream initially undergoes parallel processing wherein the item's physical and, perhaps, semantic codes are made manifest. These codes are fragile and, unless consolidated immediately, disintegrate or are overwritten by the codes of the trailing items. When an item is flagged as a target (e.g., by its physical or its semantic characteristics), an attentional gate opens, and the item is admitted into a temporary buffer whence a second, serial-processing stage ensues. Here, the item's codes are consolidated and its identity recovered and lodged in short-term memory (STM) for later recall. But upon admitting the item, the gate fails to shut immediately, thereby allowing temporally proximate items also to gain entry. This swells the buffer, placing more demands on the serial-processing stage and, thereby, making it more protracted. The crux of the two-stage model lies in the duration of this stage: Variables that impede $\mathrm{T} 1$ processing should prolong it. 
For example, if the distractors, inadvertently admitted into the buffer, are all potential target candidates, the selection and consolidation of the target codes could be more onerous, thereby prolonging processing duration. T1 processing must be completed and the item's identity lodged in STM before a new target can undergo stagetwo processing. Thus, when T2 appears, its codes would not be subject immediately to stage-two processing. Since these initial codes are fragile, a delay may jeopardize the recovery of T2's identity. Thus, according to the twostage model, what limits an immediate attentional disengagement and shift to a new locus is the processing demands of the previous target. A similar model, proposed by Jolicœur and his associates (e.g., Jolicœur, 1998, 1999; Jolicœur \& Dell'Acqua, 2000), identifies the postperceptual stage at which the sensory/perceptual codes are consolidated in STM as the point of the bottleneck.

Although an important role of attention appears to be enhancing the neural representation of the selected object so that the latter's processing may be facilitated (Kastner \& Ungerleider, 2000), target processing and attentional control are logically distinct functions. Sperling and Weichselgartner's (1995) episodic theory of attention distinguishes between the two. Using the spotlight metaphor introduced by Posner (1980), they likened attentional engagement to a spotlight's being turned on so that the action occurring under it can be processed. The critical question is the following: How long must the spotlight stay in one position before it can be shifted to illuminate somewhere else? In Sperling and Weichselgartner's conception, the point of turning on the spotlight appears to be to discover what is under the spotlight, and in this respect, the episodic model of attention bears similarities to the two-stage model (Chun \& Potter, 1995).

Raymond et al. (1992) $)^{1}$ offered a slightly different view. As in Chun and Potter (1995), this model also appeals to an attentional gate that controls entry to an identification stage. Upon engaging T1, the gate "shuts and locks" when the system senses that if more items were allowed into the identification stage, more confusion would be created (e.g., binding errors). In this locked state, attention cannot shift and engage a new target. According to this model, it is not the processing of T1 that prohibits attentional disengagement; rather, the "blink" is almost the visual system's deliberate strategy for evading binding errors by delaying attentional engagement of a new item. From this angle, the shut-and-lock model shares some similarity with the model proposed by Duncan and his associates (Duncan, Ward, \& Shapiro, 1994; Ward, Duncan, \& Shapiro, 1996), which argues that attention "dwells" for some prespecified time at a target location before it may be shifted elsewhere. They stressed that what determines the dwell time is not target processing.

\section{Varying Target and Distractor Contrast}

There have been several recent attempts at testing whether the delay in passing attentional control to T2 indeed lies in the bottleneck created by $\mathrm{T} 1$ processing. A common strategy is to vary T1 task difficulty or complexity (Chun \& Potter, 1995; Duncan et al., 1994; McLaughlin, Shore, \& Klein, 2001; Raymond, Shapiro, \& Arnell, 1995). The assumption is that the more difficult or complex the task, the longer its processing should take, and the $\mathrm{AB}$ would be larger as a consequence. $\mathrm{T} 1$ performance has been used as the manipulation check. But whether the manipulations successfully prolong processing duration ${ }^{2}$ has not been directly tested.

A different approach has been adopted for this set of experiments. The main manipulation was the luminance of the targets. Assume that the early stages of processing involve computing the perceptual code from sensory traces (Jolicœur, 1998). The target's contrast is likely to affect how quickly the sensory codes can be established in a form suitable for perceptual coding to commence. Thus, by manipulating target luminance, it is possible to influence the strength of the early sensory codes and, thus, the time course of early perceptual processing. In terms of the two-stage model (Chun \& Potter, 1995), the luminance manipulation will influence not the duration, but the onset, of Stage 2 processing.

There is good evidence that a target's contrast affects processing efficiency. Detection and identification performance is a monotonically increasing function of contrast (Graham, 1989). Reaction time to a spatial pattern decreases as its contrast increases (Ejima \& Ohtani, 1987; Gish, Shulman, Sheehy, \& Leibowitz, 1986; Lupp, Hauske, \& Wolf, 1976; Murray \& Plainis, 2003; Plainis \& Murray, 2000; Thompson \& Drasdo, 1989), implying that increases in contrast allow faster information accumulation and/or improve processing efficiency. The neurophysiological evidence accords well with the psychophysical data. The output of visual neurons increases as the contrast of a pattern increases (Albrecht \& Hamilton, 1982; Reich, Mechler, \& Victor, 2001; Tolhurst \& Movshon, 1975). Visually evoked potential latencies decrease with contrast increase (Musselwhite \& Jeffreys, 1985). Similarly, ganglion cell response latency also decreases when contrast increases (Donner, 1989).

The aim of this set of experiments was to elucidate the relationship between the $\mathrm{AB}$ and a target's contrast. But before describing the experiments, a possible side effect arising from the luminance manipulations will be considered. In these experiments, the targets and distractors were defined by different luminance (they had different contrast polarities). The RSVP sequence began with a series of distractor frames. Now, when a target appeared, the luminance of the display changed suddenly. This change, more marked when the target had a higher contrast, may have caused attention to be recruited exogenously to the temporal locus of the stimulus - that is, an orienting response (OR; Näätänen, 1992; Sokolov, 1960) may have been triggered. There is evidence that the OR increases with stimulus intensity (Turpin, Schaefer, \& Boucsein, 1999). When the features of the stimulus are not programmed into the observer's attentional control setting (ACS; Folk \& Remington, 1998; Folk, Reming- 
ton, \& Johnston, 1992, 1993), this constitutes an attentional capture. But when the stimulus is a target (i.e., its features are listed in the ACS), attentional control may be considered endogenous. The point here is that even in the case of endogenous attentional control, some aspects of the orienting could derive from exogenous control.

Experiment 1, which employed a capture paradigm, was designed to evaluate the exogenous-orienting component of the $\mathrm{AB}$. The stimuli (including the target) were letters. On some trials, a stimulus consisting of five spots (the singleton, inasmuch as its features were unique) was inserted into the stream before the target appeared. To the extent that attention was detained at the singleton's locus, capture could be deemed to have occurred. If the target appeared soon afterward, attentional engagement at its locus would be delayed somewhat, since attention had to disengage first from the singleton - that is, an $\mathrm{AB}$ would be manifest. The luminance of the singleton was manipulated. The question was whether the capture effects would be modulated by the singleton's contrast. If capture occurred, the variation in the magnitude of the $\mathrm{AB}$ for the different luminance conditions may be considered a first approximation of the dwell time due to exogenous orienting.

\section{EXPERIMENT 1A}

Raymond et al. (1992, Experiment 2) compared the experimental condition, in which observers first identified a singleton (a white letter among other black distractors) and then detected a prespecified target (a black $\mathrm{X}$ ), with the control condition, in which they ignored the white letter but merely detected the black $X$. The $A B$ was observed in the experimental condition, but not in the control condition, suggesting that the observers successfully evaded attentional capture by the single white letter. We note the following about their experiment: The two conditions were blocked, and the white letter appeared in every trial. Making the singleton's appearance predictable could have inadvertently provided immunity against capture. In this experiment, the singleton appeared only in $50 \%$ of the trials.

\section{Method}

Participants. The observers were 20 students recruited from the psychology undergraduate pool, who participated for course credit. All had normal or corrected-to-normal vision. In this and the other experiments, they were motivated by a promise of a cash bonus if their performance exceeded a modest criterion. (The criterion was varied across experiments, so that approximately $80 \%$ of the participants received the bonus.)

Apparatus. A Power Macintosh 7600 controlled the presentation of the stimuli on an Apple 15-in. multisync monitor. Stimulus presentation was synchronized to the monitor's $75-\mathrm{Hz}$ vertical refresh, using VideoToolbox routines (Pelli \& Zhang, 1991). The participants viewed the display freely from approximately $50 \mathrm{~cm}$ away. The letters were printed in a 48-point Helvetica font (subtending a visual angle of $<1^{\circ}$ ).

Procedure. The stimuli in the RSVP sequence were 24 letters (O and I excluded), with each of the 24 letters appearing as the target with approximately equal frequency. The five spots making up the singleton were arranged so that four spots formed the corners of an imaginary rectangle and the fifth was located in the center. The spots occupied roughly the same spatial extent as the letters. The distractor sequences were created as follows. The target letter was predetermined and then removed from the letter set. The order of the remaining 23 letters was shuffled. The position of the singleton (when it appeared) was randomly chosen from Frames 6-10. The target was then inserted into the frame specified by the lag condition. The singleton-absent trials were identical, except that a distractor replaced the singleton in the RSVP stream. The sequence ended four frames after the target. For the first and last frames, the $\S$ symbol was presented for approximately $250 \mathrm{msec}$. For the rest, the stimulus onset asynchrony (SOA) between two frames was approximately $93 \mathrm{msec}$. The stimulus appeared for $53 \mathrm{msec}$, followed by a blank of $40 \mathrm{msec}$. The observers were briefed to identify the single white target and to ignore everything else. When the RSVP sequence halted, a dialog box appeared, and the observers entered the identity of the target. After their response had been logged, the target identity was revealed, thereby providing feedback.

The three main variables were (1) the presence or absence of the singleton in the RSVP sequence, (2) the lag between the singleton and the target (lags 1-5, where lag 1 means the target appeared immediately after the five spots, and so on), and (3) the luminance ${ }^{3}$ of the singleton $\left(10,30\right.$, or $\left.50 \mathrm{~cd} / \mathrm{m}^{2}\right)$, which made it either the same as or darker than the distractors. The variables were factorially crossed, making up 30 trials in each block. The target letter was $90 \mathrm{~cd} / \mathrm{m}^{2}$ (white), and the distractor letters, $50 \mathrm{~cd} / \mathrm{m}^{2}$ (dark gray). The background was $70 \mathrm{~cd} / \mathrm{m}^{2}$. Although the stimuli were described in terms of their luminance, the critical variable was their contrasts, defined as the ratio of amplitude of the stimulus to mean luminance. ${ }^{4}$ The relative contrasts of the stimuli for this and the other experiments may be found in Table 1. In this report, the notations contrast ${ }^{+}$and contrast $^{-}$are used to refer to a stimulus whose luminance is, respectively, larger ("whiter") or smaller ("blacker") than the background luminance. Here, the target had contrast ${ }^{+}$, whereas the singleton and distractors had contrast ${ }^{-}$. The observers were told to focus on the single white letter and to ignore all else.

The experiment was conducted in a dark room where the only source of light came from the monitor. The participants went through 12 blocks. The 1 st block was treated as practice, and its data were not analyzed.

\section{Results}

Target identification accuracy as a function of singletontarget lag is presented in Figure 1 (left panel). The Ushaped lag functions showed that (1) the $\mathrm{AB}$ was manifest, suggesting that capture occurred, and (2) the blink became more severe as the contrast of the singleton increased. As contrast increased, the function bottomed out at a farther lag position.

To establish that capture obtained for all three contrast conditions, performance for each condition was compared with that in the singleton-absent trials. For the lowcontrast condition, a 2 (trial type: singleton present vs. absent) $\times 5$ (lags) showed that, overall, the singleton's presence did not produce significantly worse performance $\left[F(1,19)=3.382, M S_{\mathrm{e}}=0.054, p<.09\right]$. However, the effect of lag and the trial type $\times$ lag interaction were reliable $\left[F(4,76)=5.130, M S_{\mathrm{e}}=0.010, p<.001\right.$, and $F(4,76)=8.120, M S_{\mathrm{e}}=0.001, p<.001$, respectively]. At lag 1, the singleton's appearance improved performance $\left[F(1,19)=9.110, M S_{\mathrm{e}}=0.002, p<.01\right]$. This effect is akin to lag 1 sparing (Potter, Chun, Banks, \& Muckenhoupt, 1998). Performance for the singleton- 
Table 1

Luminance Parameters Used in the Experiments and Some Summary Results

\begin{tabular}{|c|c|c|c|c|c|c|}
\hline & Luminance $^{\mathrm{a}}$ & Contrast $^{\mathrm{b}}$ & $\mathrm{AB}^{\mathrm{c}}$ & Dwell Time ${ }^{c}$ & Inversion $^{\mathrm{d}}$ & Sparinge \\
\hline \multicolumn{7}{|c|}{ Experiment 1A } \\
\hline $\mathrm{H}$ & 10 & 0.750 & 70 & 2.10 & & 0.000 \\
\hline $\mathrm{M}$ & 30 & 0.400 & 46 & 1.35 & & 0.054 \\
\hline $\mathrm{L}$ & 50 & 0.167 & 36 & 1.05 & & 0.195 \\
\hline Target & 90 & 0.125 & & & & \\
\hline Distractor & 50 & 0.167 & & & & \\
\hline Background & 70 & & & & & \\
\hline \multicolumn{7}{|c|}{ Experiment 1B } \\
\hline $\mathrm{H}$ & 90 & 0.500 & 74 & 1.91 & & 0.202 \\
\hline $\mathrm{M}$ & 70 & 0.400 & 42 & 1.48 & & 0.202 \\
\hline $\mathrm{L}$ & 50 & 0.250 & 23 & 0.91 & & 0.170 \\
\hline Target & 10 & 0.500 & & & & \\
\hline Distractor & 50 & 0.250 & & & & \\
\hline Background & 30 & & & & & \\
\hline \multicolumn{7}{|c|}{ Experiment $2 \mathrm{~A}$} \\
\hline H (T1) & 10 & 0.750 & 202 & 5.05 & 0.03 & 0.105 \\
\hline $\mathrm{M}(\mathrm{T} 1)$ & 30 & 0.400 & 138 & 4.14 & 0.06 & 0.140 \\
\hline $\mathrm{L}(\mathrm{T} 1)$ & 50 & 0.167 & 114 & 3.38 & 0.12 & 0.189 \\
\hline $\mathrm{T} 2$ & 50 & 0.167 & & & & \\
\hline Distractor & 90 & 0.125 & & & & \\
\hline Background & 70 & & & & & \\
\hline \multicolumn{7}{|c|}{ Experiment 2B } \\
\hline H (T1) & 10 & 0.750 & 142 & 3.53 & & \\
\hline L (T1) & 50 & 0.167 & 137 & 3.18 & & \\
\hline $\mathrm{T} 2$ & 50 & 0.167 & & & & \\
\hline Distractor & 120 & 0.263 & & & & \\
\hline Background & 70 & & & & & \\
\hline \multicolumn{7}{|c|}{ Experiment 3} \\
\hline H (T2) & 10 & 0.750 & 13 & 0.35 & 0.05 & 0.048 \\
\hline $\mathrm{M}(\mathrm{T} 2)$ & 30 & 0.400 & 57 & 1.65 & 0.06 & 0.252 \\
\hline L (T2) & 50 & 0.167 & 142 & 3.55 & 0.14 & 0.330 \\
\hline $\mathrm{T} 1$ & 50 & 0.167 & & & & \\
\hline Distractor & 90 & 0.125 & & & & \\
\hline Background & 70 & & & & & \\
\hline \multicolumn{7}{|c|}{ Experiment 4A } \\
\hline H (T1\&T2) & 10 & $0.750(6.00)$ & 5 & 0.17 & 0.13 & 0.065 \\
\hline M (T1\&T2) & 30 & $0.400(3.20)$ & 62 & 2.00 & 0.09 & 0.224 \\
\hline L (T1\&T2) & 50 & $0.167(1.33)$ & 149 & 3.83 & 0.11 & 0.315 \\
\hline Distractor & 90 & 0.125 & & & & \\
\hline Background & 70 & & & & & \\
\hline \multicolumn{7}{|c|}{ Experiment 4B } \\
\hline H (T1\&T2) & 90 & $0.500(1.00)$ & 131 & 3.50 & 0.13 & 0.362 \\
\hline M (T1\&T2) & 70 & $0.400(0.80)$ & 155 & 3.72 & 0.12 & 0.423 \\
\hline L (T1\&T2) & 50 & $0.250(0.50)$ & 174 & 4.28 & 0.12 & 0.405 \\
\hline Distractor & 10 & 0.500 & & & & \\
\hline Background & 30 & & & & & \\
\hline \multicolumn{7}{|c|}{ Experiment 5} \\
\hline H (T1) & 10 & $0.800(5.59)$ & 140 (104) & $3.28(2.67)$ & 0.06 & 0.113 \\
\hline L (T1) & 60 & $0.200(1.40)$ & $89(47)$ & $2.22(1.78)$ & 0.20 & 0.297 \\
\hline $\mathrm{T} 2$ & 60 & $0.200(1.40)$ & & & & \\
\hline Distractor & 120 & 0.143 & & & & \\
\hline Background & 90 & & & & & \\
\hline
\end{tabular}

Note-AB, attentional blink; $\mathrm{H}$, high contrast; $\mathrm{M}$, medium contrast; $\mathrm{L}$, low contrast; $\mathrm{T} 1$, first target; T2, second target. aLuminance is given in $\mathrm{cd} / \mathrm{m}^{2}$. ${ }^{\mathrm{b}}$ Contrast is the ratio of amplitude to mean luminance (i.e., Michelson's contrast). Absolute values are reported. Relative target: distractor contrast is given in parentheses. ${ }^{c}$ For Experiments $1 \mathrm{~A}, 1 \mathrm{~B}, 2 \mathrm{~B}$, and $5, \mathrm{AB}$ is estimated from five lags; for Experiments 2A, 3, and 4, from seven lags. AB and dwell time estimates are calculated using McLaughlin, Shore, and Klein's (2001) formula. For Experiment 5, the estimates in parentheses were calculated from trials in which a blank frame was inserted in the rapid serial visual presentation sequence. dInversion estimated for T1-T2 lag 1. eT1-T2; sparing estimated by calculating the difference between lag 1 and lag 2 scores. 


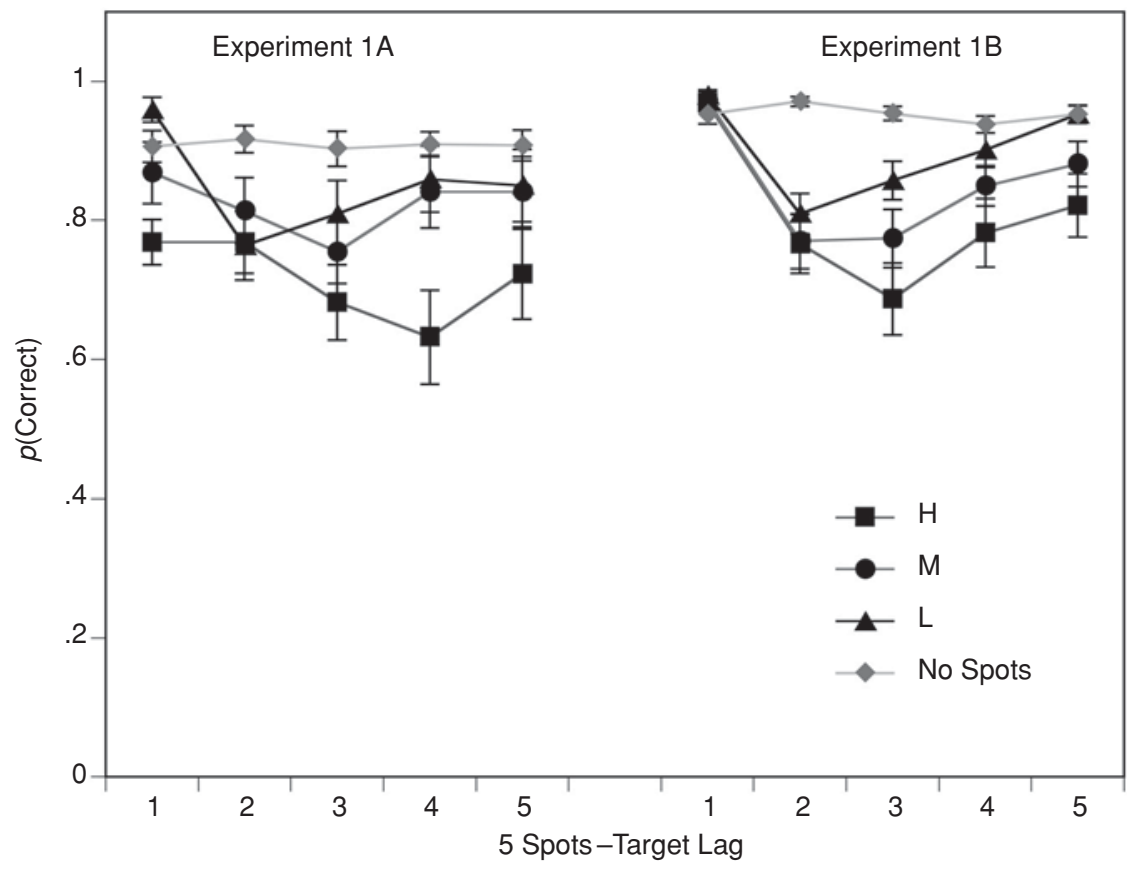

Figure 1. Experiments 1A and 1B: probability of target identification as a function of the lag between the five spots and the target. The contrast of the spots was varied: square $=$ high, circle $=$ medium, and triangle $=$ low contrast; diamond $=$ baseline. Error bars $=1 S E$.

present trials was poorer than baseline only when the target appeared two lags away $\left[F(1,19)=10.126, M S_{\mathrm{e}}=\right.$ $0.012, p<.005]$.

For the medium-contrast singleton, an analogous $2 \times$ 5 analysis of variance (ANOVA) showed reliably poorer performance for the singleton-present trials $[F(1,19)=$ $\left.5.140, M S_{\mathrm{e}}=0.070, p<.05\right]$. There was a small hint of a lag $\times$ trial type interaction $\left[F(4,76)=2.462, M S_{\mathrm{e}}=\right.$ $0.007, p<.06]$. Simple effects tests showed reliable differences only at lags 2 and $3\left[F(1,19)=5.485, M S_{\mathrm{e}}=\right.$ $0.039, p<.05$, and $F(1,19)=13.622, M S_{\mathrm{e}}=0.032, p<$ .005 , respectively]. For the high-contrast spots, capture effects were clearly demonstrated $[F(1,19)=32.999$, $\left.M S_{\mathrm{e}}=0.057, p<.001\right]$. There was no trial type $\times$ lag interaction $\left[F(4,76)=2.032, M S_{\mathrm{e}}=2.032, p>.1\right]$. Performance was worse for the singleton-present trials for all five lags. For the medium- and high-contrast conditions, there was no lag 1 sparing: Performance at lags 1 and 2 was not different. But the minima of these functions (see below) were at lags 3 and 4, respectively, and not at lag 2 (as in the other experiments in this set).

The next set of analyses examined whether the AB was modulated by the contrast of the spots. Data from the singleton-present trials were analyzed as a 3 (contrast of the five spots: high, medium, or low) $\times 5$ (lags) ANOVA. The results showed that performance declined as contrast increased $\left[F(2,38)=26.336, M S_{\mathrm{e}}=0.019\right.$, $p<.001]$. Performance also varied with singleton-target $\operatorname{lag}\left[F(4,76)=4.240, M S_{\mathrm{e}}=0.027, p<.005\right]$. The lag function was modulated by the contrast of the singleton $\left[F(8,152)=3.273, M S_{\mathrm{e}}=0.015, p<.005\right]$. Trends analysis showed that the quadratic trend was reliable and did not interact with the contrast variable $(F<1)$.

The minima of the lag functions varied with the contrast of the spots. When the contrast was low, the lag function bottomed out at lag 2. But for the medium- and high-contrast spots, their minima were at lags 3 and 4, respectively. The interpretation is that attention dwelled longer at the singleton that had a higher contrast and, as a result, the minimum of the lag function shifted laterally. To provide a formal analysis of this observation, attention dwell times for the three contrast conditions were estimated, using the algorithm proposed by McLaughlin et al. (2001; estimates for this and the other experiments may be found in Table 1). ${ }^{5}$ A one-factor ANOVA comparing the dwell time estimates ${ }^{6}$ showed an overall effect $\left[F(2,38)=6.357, M S_{\mathrm{e}}=0.920, p<.05\right]$. Dwell time was longer when contrast was higher. Post hoc tests showed that, as compared with the medium-luminance spots, dwell time for the high-luminance spots was longer $\left[F(1,19)=6.333, M S_{\mathrm{e}}=0.444, p<.05\right]$. There was no reliable difference between the medium and the low conditions $(F \approx 1)$. An analogous ANOVA using AB magnitude (Table 1) as the dependent variable showed an overall contrast effect $\left[F(2,38)=5.524, M S_{\mathrm{e}}=1,125, p<\right.$ $.01]$. The blink magnitude was larger when contrast was higher. Post hoc tests showed a larger AB for the highcontrast singleton than for a medium-contrast one 
$\left[F(1,19)=5.463, M S_{\mathrm{e}}=533, p<.05\right]$. There was no difference between the medium- and the low-contrast conditions $(F<1)$.

\section{EXPERIMENT 1B}

A target appearing after a five-spot singleton that had a higher contrast was identified more poorly than it was when a lower contrast singleton preceded it. The poorer performance could be attributed to forward masking by the singleton, with the potency of the forward mask increasing with contrast. Although there probably must have been some measure of masking, this explanation is not entirely satisfactory, because the masking effects would have to last more than $500 \mathrm{msec}$, occurring even when several distractors intervened between the putative mask and the target. Also, for the high-contrast condition, the forward-masking effect would have to be smaller for an immediately trailing target than for one that appeared $400 \mathrm{msec}$ later. Nor can this explanation account for why performance was poorer even when the singleton and the distractors had the same contrast. Nevertheless, to test the masking hypothesis, a different range of contrasts was manipulated. The background luminance was $30 \mathrm{~cd} / \mathrm{m}^{2}$. The distractors were $50 \mathrm{~cd} / \mathrm{m}^{2}$, and the single target $10 \mathrm{~cd} / \mathrm{m}^{2}$. As in the previous experiment, there were three luminance values for the five spots: 90 , 70 , and $50 \mathrm{~cd} / \mathrm{m}^{2}$. This time, the singleton had contrast ${ }^{+}$, and the target contrast ${ }^{-}$, reversing the contrast polarities in Experiment 1A. In addition, these different contrast values allowed a test of the generality of the results of the previous experiment. The range of contrasts was narrower than in the previous experiment (see Table 1).

The design and procedure were identical to those in Experiment 1A. Another 21 observers from the same pool participated.

\section{Results}

The data (see Figure 1, right panel) were treated in the manner described previously. In general, the results not only replicated those in Experiment 1A, but also provided clearer evidence of attentional capture. When the luminance of the singleton was identical to that of the distractors (i.e., low contrast), performance was reliably worse than in the baseline condition. A 2 (singleton present or absent) $\times 5$ (lags) ANOVA showed an overall advantage for the baseline condition $[F(1,20)=14.909$, $\left.M S_{\mathrm{e}}=0.010, p<.001\right]$. There was a singleton presence $\times$ lag interaction $\left[F(4,80)=12.276, M S_{\mathrm{e}}=0.005\right.$, $p<.001]$. Simple effects tests showed that the singleton produced poorer performance only for lags 2 and 3 $\left[F(1,20)=28.275, M S_{\mathrm{e}}=0.005, p<.001\right.$, and $F(1,20)=$ 11.766, $\left.M S_{\mathrm{e}}=0.004, p<.005\right]$. Analogous ANOVAs for the medium- and the high-contrast conditions yielded comparable results.

As in Experiment 1A, higher contrast spots led to worse target recovery. A 3 (contrasts) $\times 5$ (lags) ANOVA showed a reliable contrast effect $[F(2,40)=15.726$,
$\left.M S_{\mathrm{e}}=0.015, p<.001\right]$. Performance varied as a function of singleton-target lag $\left[F(4,80)=16.857, M S_{\mathrm{e}}=\right.$ $0.025, p<.001]$. The lag effect was modulated by contrast of the singleton $\left[F(8,160)=2.416, M S_{\mathrm{e}}=0.01\right.$, $p<.05]$. Further analyses, using dwell time and AB magnitudes as dependent variables, provided consistent results.

In Experiment 1A, the singleton contrast modulated lag 1 performance, and an explanation based on forward masking of the target by the singleton was proposed. Here, lag 1 performance (the point at which masking would have been most potent) was the same for all three contrast conditions, suggesting that forward masking could not have been critical.

\section{Discussion: Experiments 1A and 1B}

There are three main results in Experiment 1. First, attention was captured at the (temporal) site of the singleton, and this triggered an $\mathrm{AB}$. Second, the magnitude of the $\mathrm{AB}$ was directly related to the contrast of the spots. Pooled across the two experiments, the correlation $(r)$ between contrast of the spots and the magnitude of the AB is .86 $(p<.05)$. Third, dwell times also increased with contrast of the spots (the corresponding correlation is .95, $p<.005$ ). Could these results be explained by appealing to forward masking of the target by the five spots? Some measure of masking probably occurred for the shortest lag (possibly in Experiment 1A, where the contrast of the target was lower than that of the singleton), but this cannot be a critical factor, since performance was best when the target appeared immediately after the five spots, when forward masking ought to be most potent (Breitmeyer, 1984). The AB was obtained even when the contrast of the spots was lower than that of the target (Experiment 1B) and extended more than $400 \mathrm{msec}$ (the longest lag tested) where several distractors had intervened between the singleton and the target.

According to the contingent capture hypothesis (Folk \& Remington, 1998; Folk, et al., 1992, 1993), the potency of a stimulus in capturing attention depends critically on the observer's ACS. In this experiment, the singleton occupied the same spatial extent as the other letters, and so its appearance would not constitute an onset. The target's contrast polarity was the opposite of the distractors'. The singleton's luminance either was identical to or had the same contrast polarity as that of the distractors. It needs to be emphasized that the target's luminance was constant across trials (thus, there was no uncertainty). Since the singleton possessed neither the features (five spots) nor the luminance profile of the target, attention capture should not obtain. Perhaps one could simply program into the ACS an instruction to attend to any change in mean luminance. But this would still predict that when the singleton and the distractors had the same contrast, capture ought not to be observed, and no $\mathrm{AB}$ should be manifest. What is noteworthy here is not that the effects were small, but that capture occurred at all. 
The results extended a recent finding of Folk, Leber, and Egeth (2002), who also found capture, using an RSVP procedure. But Folk et al.'s experiment demonstrated capture at a location that was spatially distinct from that of the target. The AB that they found probably consisted of two separate effects: attention dwell at the capture site and the spatial shift from the capture site back to the target location. In this experiment, the target and the singleton occupied the same location, obviating the need for an additional spatial shift. The delay observed would be due entirely to attentional engagement by the singleton.

Attentional control by the singleton was exogenous; what was observed could be the effects of a blink caused by an OR to an unexpected stimulus (Näätänen, 1992; Sokolov, 1960). When the high-contrast spots appeared, there was a larger change in contrast, making the spots informationally more "novel" than when the contrast of the spots was more similar to that of the distractors. There is recent evidence that novel stimuli produce larger N2-P3 amplitudes and longer viewing durations (Daffner et al., 1998). It has also been shown that the intensity of the stimulus affects the OR (Turpin et al., 1999). This direct relationship between the singleton's contrast and the magnitude of the AB may, however, be specific to exogenous attentional control. Since the mechanisms underlying endogenous and exogenous attentional control may be different (e.g., LaBerge, 2002), it is entirely possible that when attentional control is endogenous, the effect of stimulus contrast on $\mathrm{AB}$ can be different. The next experiment addressed this issue.

\section{EXPERIMENT 2A}

In Experiment 1, the singleton's contrast modulated the magnitude of the AB. The aim of Experiment 2 was to examine whether comparable results would obtain when attentional control was endogenous. Specifically, the question was the following: What is the effect of T1 contrast on T2 recovery? Stimulus processing improves as contrast increases (Ejima \& Ohtani, 1987; Gish et al., 1986; Graham, 1989; Lupp et al., 1976). If stimulus processing determines attentional dwell time, a high-contrast $\mathrm{T} 1$ should allow an earlier attention disengagement from $\mathrm{T} 1$ and should produce a smaller $\mathrm{AB}$.

\footnotetext{
Method

Procedure. The participants were required to identify two letter targets. The two main variables were (1) the luminance of T1 (10, 30 , or $50 \mathrm{~cd} / \mathrm{m}^{2}$, contrast ${ }^{-}$) and (2) the lag between the two targets (lags 1-7). T2's luminance was $50 \mathrm{~cd} / \mathrm{m}^{2}$, and that of the distractors was $90 \mathrm{~cd} / \mathrm{m}^{2}$. The background was $70 \mathrm{~cd} / \mathrm{m}^{2}$. In this and the other experiments, the observers were shown examples of letters printed in the three target luminance values during the preview. They were told explicitly to "look for the dark letters; ignore the white ones."

The sequences were constructed as described previously. After the observers had lodged their responses, the target identities were revealed. The participants were 21 new observers recruited from the same pool. They went through 13 blocks of 21 trials each. Data from the 1st practice block were excluded from analysis.
}

\section{Results and Discussion}

The data are presented in Figure 2 [left plot, $p(\mathrm{~T} 1)$; right plot, $p(\mathrm{~T} 2 \mid \mathrm{T} 1)]$. Identification performance of T1 improved with contrast. A high-contrast T1 $(M=.986$, $S D=.03$ ) was identified significantly better than a medium-contrast one $[M=.972, S D=.04 ; F(1,20)=$ $\left.7.356, M S_{\mathrm{e}}=0.002, p<.05\right]$, which in turn was identified better than a low-contrast T1 $[M=.913, S D=0.09$; $\left.F(1,20)=23.023, M S_{\mathrm{e}}=0.01, p<.001\right]$. The critical finding here is that better $\mathrm{T} 1$ performance paradoxically produced worse $\mathrm{T} 2$ performance. The discussion for the rest of this section is organized around key features of the results.

T1 contrast and $T 2$ performance. The right plot (Figure 2), where the dependent variable was $p(\mathrm{~T} 2 \mid \mathrm{T} 1)$, showed clearly that (1) the $\mathrm{AB}$ obtained for all three contrasts but (2) T1 contrast modulated performance. A 3 (contrast) $\times 7$ (lags) ANOVA showed an overall main effect of T1 contrast $\left[F(2,40)=10.852, M S_{\mathrm{e}}=0.023, p<\right.$ $.001]$. Performance also varied with lags $[F(6,120)=$ $\left.26.478, M S_{\mathrm{e}}=0.046, p<.001\right]$. Critically, performance across lags was modulated by $\mathrm{T} 1$ contrast $[F(12,240)=$ 5.364, $\left.M S_{\mathrm{e}}=0.015, p<.001\right]$. By lag 3, differences among the contrast conditions disappeared.

Effect of $T 1$ contrast on $A B$ magnitude and dwell time. To further examine the effect of T1 contrast, two separate indices were computed: (1) the size of the blink and (2) the minimum lag (dwell time) before performance returned to baseline ${ }^{7}$ (see Table 1). A one-way ANOVA showed that the magnitude of the blink increased with T1 contrast $\left[F(2,40)=16.114, M S_{\mathrm{e}}=2,722, p<\right.$ $.001]$. The blink was significantly larger when $\mathrm{T} 1 \mathrm{con}$ trast was high, as compared with the medium condition $\left[F(1,20)=20.537, M S_{\mathrm{e}}=1,067, p<.001\right]$. The difference between the medium- and the low-contrast conditions was, however, not reliable $\left[F(1,20)=3.357, M S_{\mathrm{e}}=\right.$ $878, p<.09]$.

An analogous one-way ANOVA in which the dwell time estimates were used as the dependent variable yielded clearer results. Dwell times were prolonged as T1 contrast increased $\left[F(2,40)=11.992, M S_{\mathrm{e}}=1.219, p<\right.$ .001]. Paired comparisons showed that dwell time was longer when $\mathrm{T} 1 \mathrm{had}$ a high, rather than a medium, contrast $\left[F(1,20)=10.169, M S_{\mathrm{e}}=0.423, p<.005\right]$. A medium-contrast $\mathrm{T} 1$ led to longer dwell times than did a low-contrast T1 $\left[F(1,20)=5.322, M S_{\mathrm{e}}=0.573, p<\right.$ $.05]$.

The magnitudes of the $\mathrm{AB}$ and dwell times were much larger here than in Experiment 1A, although the same luminance values were used in both experiments (see Table 1). This pattern of results is consistent with Jolicœur's (1999) view that adding the short-term consolidation phase for T1 (required in Experiment 2, but not in Experiment 1A) prolongs dwell time.

OR and dwell time. In Experiment 1, the longer dwell time at the locus of a high-contrast singleton was attributed to a bigger OR, because the singleton's appearance 


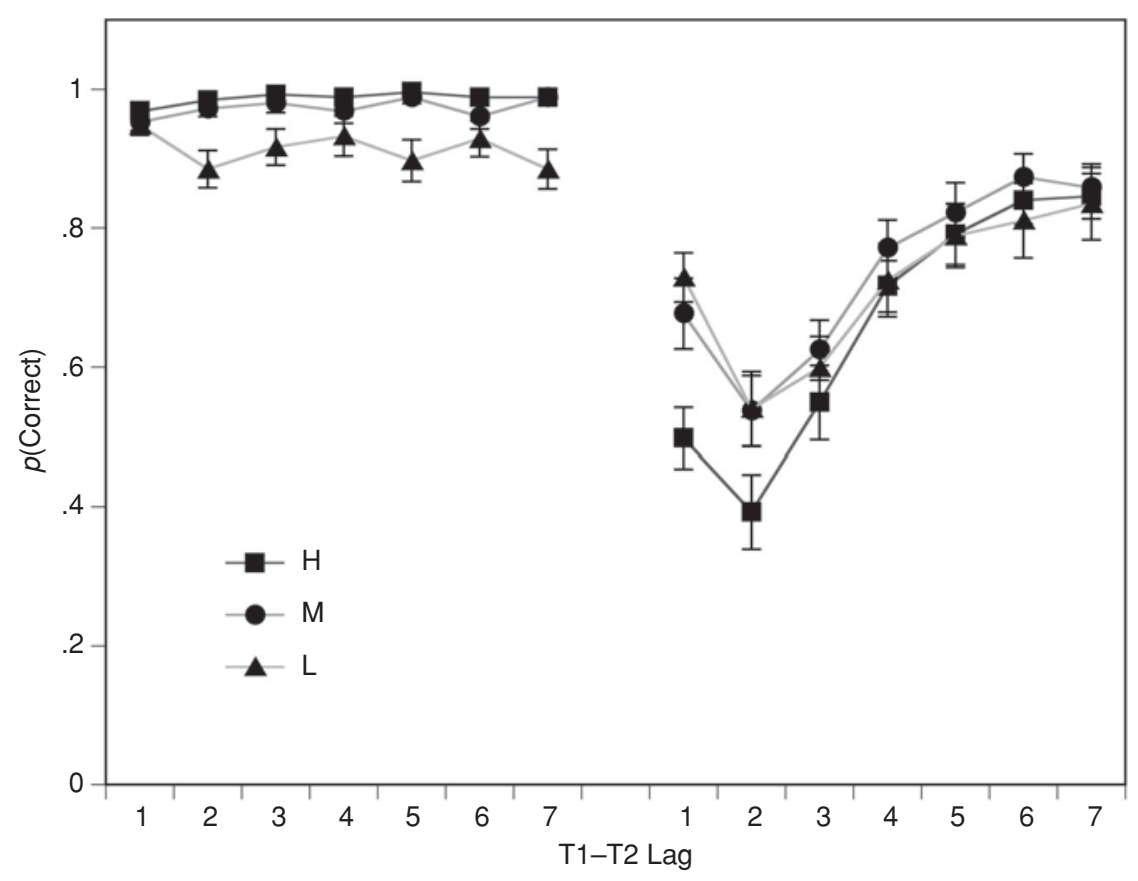

Figure 2. Experiment 2A: identification performance as a function of T1-T2 lag. Left panel, $p(\mathrm{~T} 1)$; right panel, $p(\mathrm{~T} 2 \mid \mathrm{T} 1)$. The contrast of $\mathrm{T} 1$ was varied: square $=$ high, circle $=$ medium, and triangle $=$ low contrast. Error bars $=1 S E$.

would have led to the bigger change in the mean luminance of the display. Although the context of Experiment 2 was different (T1's luminance would have been programmed into the observers' ACS, for example), there should still be an OR component, since T1's appearance produced a change in mean luminance, with a larger change when $\mathrm{T} 1 \mathrm{had}$ a higher contrast. The question is whether the longer dwell time found for a high-contrast T1 was due solely to the larger OR that it engendered.

The absolute performance in the two experiments cannot be directly compared, because they had different task requirements. But an indirect comparison can be made of the differences between the high- and the low-contrast conditions in the two experiments. Assume that the locus of the effect of contrast is in perceptual processing, and not in the postperceptual consolidation of T1 (e.g., Jolicœur, 1999). If the costs of a longer dwell time were due entirely to the OR, the difference between the highand the low-contrast conditions in the two experiments should be approximately the same. In Experiment 1A, the difference in dwell times between the high and the low conditions was approximately 1 lag (difference in $\mathrm{AB}=34$ ). In Experiment 2, the difference in dwell times grew almost to 1.7 lags (difference in $\mathrm{AB}=88$ ). ${ }^{8} \mathrm{In}$ deed, if we expect that there should be facilitation when T1 contrast is increased, processing duration should shorten. This means that the difference between the highand the low-contrast conditions should, in fact, shrink in Experiment 2, because the processing facilitation from a higher contrast T1 ought to compensate for the larger OR that it evoked.
The results contradicted this prediction. Thus, the faster processing of a higher contrast target paradoxically produced a larger, and not a smaller, $\mathrm{AB}$. There are two conclusions: (1) The longer dwell times cannot be attributed solely to longer exogenous orienting to a higher contrast T1, and (2) faster T1 processing did not produce shorter dwell times.

In summary, the main results are as follows: (1) The accuracy of identifying T1 varied directly with contrast, and (2) the magnitude and duration of the blink also varied directly with $\mathrm{T} 1$ contrast. The results of $\mathrm{T} 1$ performance agree with extant psychophysical data, which have consistently shown that the higher the contrast, the shorter the latencies (Ejima \& Ohtani, 1987; Gish et al., 1986; Lupp et al., 1976; Thompson \& Drasdo, 1989). As T1's contrast increased, its processing was facilitated. But paradoxically, attention appeared to dwell longer. The entire argument so far hinges on the claim that processing time for a higher contrast target was shorter. This claim was tested directly in Experiment 2B.

\section{EXPERIMENT 2B}

The paradigm was a variation of Jolicœur (1999): a speeded detection task, combined with a nonspeeded identification task. There were two targets. The first was a five-spot configuration (as in Experiment 1). Observers had to "detect" the spots by striking a prespecified key as quickly as possible. The second target was a letter, which they had to identify (this task was not speeded). A simple reaction time task for $\mathrm{T} 1$ was chosen, because 
what was of interest was the effect of target luminance on perceptual processing. Jolicœur showed that the demands of postperceptual processing (e.g., response selection difficulty) modulated the $\mathrm{AB}$.

\section{Method}

There were two main variables: (1) the luminance of the five spots (high $=10 \mathrm{~cd} / \mathrm{m}^{2}$ and low $=50 \mathrm{~cd} / \mathrm{m}^{2}$, contrast $\left.{ }^{-}\right)$and (2) the lag between T1 and T2 (lags 2-6). The background was $70 \mathrm{~cd} / \mathrm{m}^{2}$, and the distractors $120 \mathrm{~cd} / \mathrm{m}^{2}$. The luminance of T2 was $50 \mathrm{~cd} / \mathrm{m}^{2}$. In each block, there were 10 ( 2 contrasts $\times 5$ lags $)$ trials containing the spots. In addition, there were 5 catch trials where the spots were not presented. The RSVP sequences were created in the manner described previously. Each of the 18 new participants, recruited from the same pool, went through 15 blocks of trials.

\section{Results and Discussion}

The data from 1 observer were excluded because of poor detection accuracy $(<80 \%)$. The mean response times for the high- and the low-contrast spots were $391 \mathrm{msec}(S D=76)$ and $416 \mathrm{msec}(S D=92)$, respectively. The 25 -msec difference was reliable $[F(1,16)=$ $\left.14.928, M S_{\mathrm{e}}=346, p<.001\right]$. The data, probability of "detecting" the spots, $p$ (T1), and the probability of identifying T2 for trials in which T1 was detected, are presented in Figure 3 [left plot, $p(\mathrm{~T} 1)$; right plot, $p(\mathrm{~T} 2 \mid \mathrm{T} 1)$ ]. There was no difference between the high- and the lowcontrast conditions for either the T1 or the T2 data. Separate ( 2 luminance $\times 5$ lags) ANOVAs bore this out. For the $\mathrm{T} 1 \mathrm{data}$, all the effects were not significant $\left(F_{\mathrm{S}}<1\right)$. For the $\mathrm{T} 2$ data, only the lag effect was reliable $[F(4,64)=$ $\left.22.82, M S_{\mathrm{e}}=0.044, p<.001\right]$.
Response to a high-contrast T1 was faster. But unlike in Experiment 2A (and Experiment 5, below), T1 contrast did not modulate $\mathrm{T} 2$ performance. These seemingly discrepant results may be attributed to the different demands in the two tasks. In Experiment 2B, speed of the $\mathrm{T} 1$ response was emphasized. To achieve maximum speed, the motor program that drives the keypress must be immediately loaded when enough evidence has been accumulated to support the decision that the target has appeared. Thus, the emphasis on speed may have induced an earlier termination of the perceptual stage and the commencement of the postperceptual motor phase. But the 25-msec headstart for the high-contrast condition did not translate into better $\mathrm{T} 2$ identification.

The results of the two experiments are the following: (1) When speed was emphasized, an increase in contrast led to faster target processing, but (2) when speed was not emphasized, the same increase in contrast led to longer attention dwell times. The implication here is that processing per se does not determine how long attention is detained at a particular locus. Although processing is facilitated by a higher contrast, a rapid disengagement of attention occurred only when task instructions put a premium on response speed.

\section{EXPERIMENT 3}

An important finding in the $\mathrm{AB}$ literature is that when the RSVP sequence ends on T2, the blink is eliminated. According to the substitution-masking hypothesis (e.g., Di Lollo, Enns, \& Rensink, 2000; Giesbrecht \& Di Lollo,

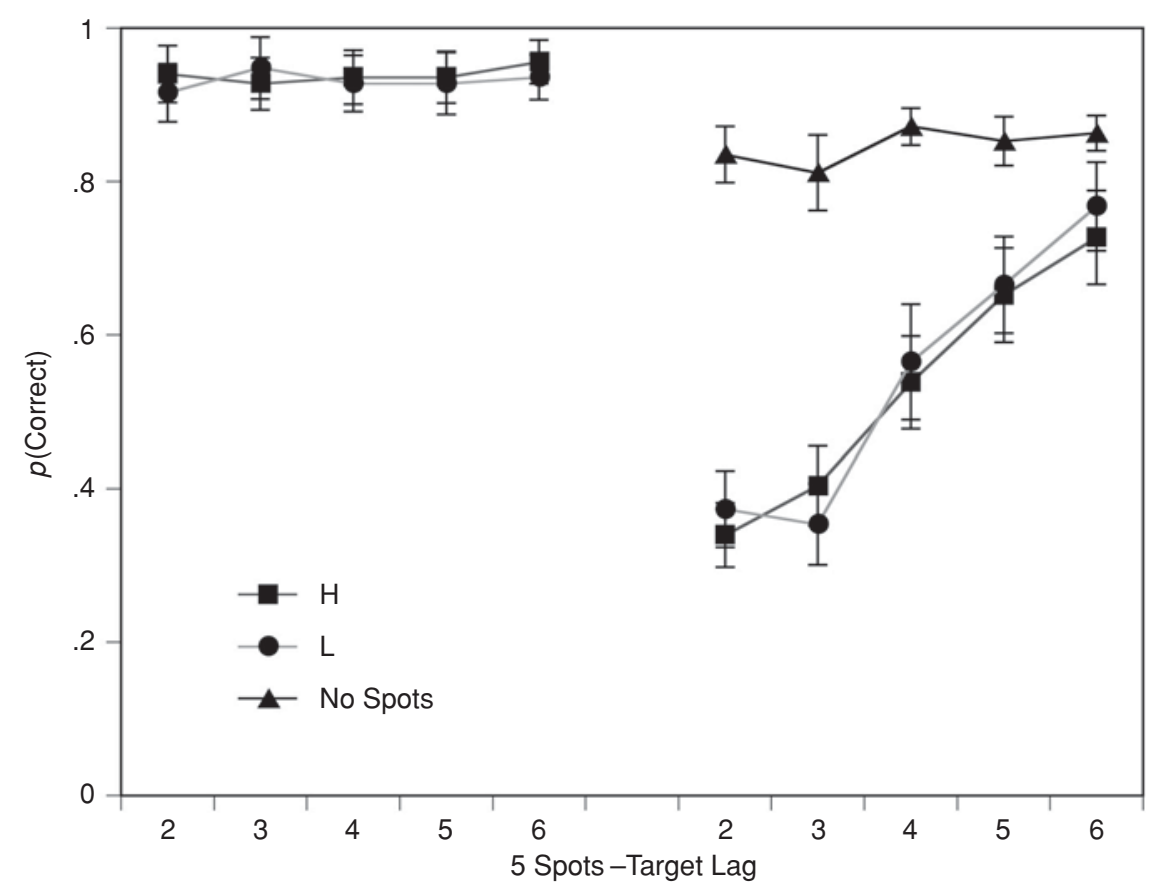

Figure 3. Experiment 2B: identification performance as a function of 5 Spots-T2 lag. Left panel, $p(\mathrm{~T} 1)$; right panel, $p(\mathrm{~T} 2 \mid \mathrm{T} 1)$. T1 was identified by a speeded keypress. The contrast of T1 was varied: square $=$ high, circle $=$ medium, and triangle $=$ low contrast. Error bars $=1 S E$. 
1998), delay in attentional engagement of $T 2$ makes the latter's codes vulnerable to replacement by the codes of the trailing distractor. In this respect, the visibility of the trailing distractor is critical. In Experiment 3, the contrast of the distractors (and therefore, T2's trailing mask) was kept constant. The contrast of T2 was varied. The question was how the contrast of T2 might modulate the masking potency of the trailing distractor.

\section{Method \\ Procedure. The design was identical to that in Experiment 2A. The background and distractor luminance was 70 and $90 \mathrm{~cd} / \mathrm{m}^{2}$, re- spectively. The luminance of T2 was varied $\left(10,30\right.$, or $50 \mathrm{~cd} / \mathrm{m}^{2}$, contrast $^{-}$), whereas the luminance of $\mathrm{T} 1$ was fixed at $50 \mathrm{~cd} / \mathrm{m}^{2}$. The observers were 20 students drawn from the same pool. There were 13 blocks, each containing 21 trials. Data from the 1 st practice block were excluded from analysis.}

\section{Results and Discussion}

The data are presented in Figure 4 [left plot, $p(\mathrm{~T} 1)$; right plot, $p(\mathrm{~T} 2 \mid \mathrm{T} 1)]$. For the lag 1 condition, T1's performance was poorer when T2's contrast was higher. Although some measure of backward masking of T1 by T2 was to be expected, an analysis of the T1-T2 conjoint probabilities showed no reliable difference among the three conditions $\left[F(2,38)=2.427, M S_{\mathrm{e}}=0.022, p>.1\right]$. This suggests that the two targets were probably recovered in a single episode, and overall, the contrast differences between $\mathrm{T} 1$ and $\mathrm{T} 2$ did not affect their recovery.

T2 identification correlated with its contrast. A 3 (contrast) $\times 7$ (lags) ANOVA confirmed that performance improved when T2's contrast increased $[F(2,38)=63.765$, $\left.M S_{\mathrm{e}}=0.029, p<.001\right]$. Performance also varied as a function of lags $\left[F(6,114)=41.354, M S_{\mathrm{e}}=0.020, p<\right.$ $.001]$. The significant interaction between the two variables $\left[F(12,228)=6.817, M S_{\mathrm{e}}=0.015, p<.001\right]$ showed that T2 contrast modulated the recovery from AB. Polynomial trend analyses revealed that the reliable quadratic trend $\left[F(1,19)=21.095, M S_{\mathrm{e}}=0.019, p<.001\right]$ was modulated by the contrast variable $[F(1,19)=18.325$, $\left.M S_{\mathrm{e}}=0.021, p<.001\right]$. When T2 contrast was high, the lag function was flatter, implying a weaker AB. Consistent with the performance results, a one-way ANOVA in which blink magnitude was used as the dependent variable showed that $\mathrm{AB}$ decreased as $\mathrm{T} 2$ contrast increased $\left[F(2,38)=45.079, M S_{\mathrm{e}}=1,914, p<.001\right]$. Planned comparisons showed that the $\mathrm{AB}$ was significantly smaller when T2 had a high, as compared with a medium, contrast $\left[F(1,19)=17.706, M S_{\mathrm{e}}=540, p<.001\right]$. The medium-contrast $\mathrm{T} 2$, in turn, produced a smaller $\mathrm{AB}$ than when it had a low contrast $\left[F(1,19)=50.665, M S_{\mathrm{e}}=\right.$ $720, p<.001]$.

These results extend Giesbrecht and Di Lollo's (1998) finding that the $A B$ disappeared when the RSVP sequence ended with T2. Here, when T2 had a higher contrast, the $\mathrm{AB}$ was drastically reduced (blink magnitude was only 15 , although the lag effect was still reliable). Di Lollo and his associates emphasized the importance of the visibility of the trailing distractor. What the Experiment 3 results showed is that the trailing distractor became less effective as a mask when T2's contrast increased. Thus, even when the trailing distractor is fully visible (contrast $=12.5 \%$ ), protection from substitution masking may be obtained by increasing $\mathrm{T} 1$ contrast.

\section{EXPERIMENT 4}

In Experiment 4, the relative contributions of $\mathrm{T} 1$ and $\mathrm{T} 2$ contrasts on $\mathrm{AB}$ were examined. In Experiment 2, the magnitude of the blink was positively correlated with $\mathrm{T} 1$ contrast. But in Experiment 3, there was a negative relationship between $\mathrm{AB}$ magnitude and $\mathrm{T} 2$ contrast. In this experiment, $\mathrm{T} 1$ and $\mathrm{T} 2$ contrasts were correlated. The question is how a larger $\mathrm{AB}$ from a prolonged dwell at a high-contrast $\mathrm{T} 1$ would be compensated by the protection against substitution masking by increasing $\mathrm{T} 2$ contrast.

\section{Method}

Procedure. Two experiments, in which different luminance values were used, were conducted. The design was a 3 (contrast levels) $\times 7$ (lags) factorial. In Experiment 4A, the background and distractor luminance was 70 and $90 \mathrm{~cd} / \mathrm{m}^{2}$, respectively. T1 and T2 luminance was 10, 30, or $50 \mathrm{~cd} / \mathrm{m}^{2}\left(\right.$ contrast $\left.^{-}\right)$. In Experiment 4B, the background was $30 \mathrm{~cd} / \mathrm{m}^{2}$, and the distractors $10 \mathrm{~cd} / \mathrm{m}^{2} . T 1$ and T2 were 90,70 , or $50 \mathrm{~cd} / \mathrm{m}^{2}$ (contrast $\left.{ }^{+}\right)$. The range of contrasts was larger in Experiment 4A (see Table 1).

The same procedure as that described previously was used. There were 12 observers for Experiment 4A and 18 for Experiment 4B. They each underwent 13 blocks of 21 trials. As before, the 1st block was treated as a practice block, and its data were excluded from analysis.

\section{Results and Discussion}

The data, $p(\mathrm{~T} 2 \mid \mathrm{T} 1)$, are presented in Figures $5 \mathrm{~A}$ and 5B (left plot, Experiment 4A; right plot, Experiment 4B). Results of the ANOVAs for the two experiments were largely consistent and reported as $F_{\mathrm{A}}$ and $F_{\mathrm{B}}$, for Experiments $4 \mathrm{~A}$ and $4 \mathrm{~B}$, respectively. The critical finding is that the magnitude of the $\mathrm{AB}$ is modulated by the relative target:distractor contrast.

Target contrast and performance. T1 performance improved with contrast $\left[F_{\mathrm{A}}(2,22)=24.654, M S_{\mathrm{e}}=0.01\right.$, $p<.001$, and $\left.F_{\mathrm{B}}(2,34)=22.919, M S_{\mathrm{e}}=0.013, p<.001\right]$. More critically, T2 performance also improved with contrast. A 3 (contrasts) $\times 7$ (lags) ANOVA showed that performance improved with target contrast $\left[F_{\mathrm{A}}(2,22)=\right.$ $48.331, M S_{\mathrm{e}}=0.043, p<.001$, and $F_{\mathrm{B}}(2,34)=45.958$, $\left.M S_{\mathrm{e}}=0.016, p<.001\right]$. Performance also varied with lags $\left[F_{\mathrm{A}}(6,66)=17.521, M S_{\mathrm{e}}=0.02, p<.001\right.$, and $\left.F_{\mathrm{B}}(6,102)=34.786, M S_{\mathrm{e}}=0.049, p<.001\right]$. The lag effect was modulated by the contrast variable $\left[F_{\mathrm{A}}(12,132)=\right.$ $5.076, M S_{\mathrm{e}}=0.012, p<.001$, and $F_{\mathrm{B}}(12,204)=2.050$, $\left.M S_{\mathrm{e}}=0.017, p<.05\right]$. The modulation of the lag effect by contrast is clearer in Experiment 4A. For the highcontrast condition, the lag function was almost flat.

T2 contrast and protection against the blink. A high-contrast $\mathrm{T} 1$ may produce a larger $\mathrm{AB}$, but this was 


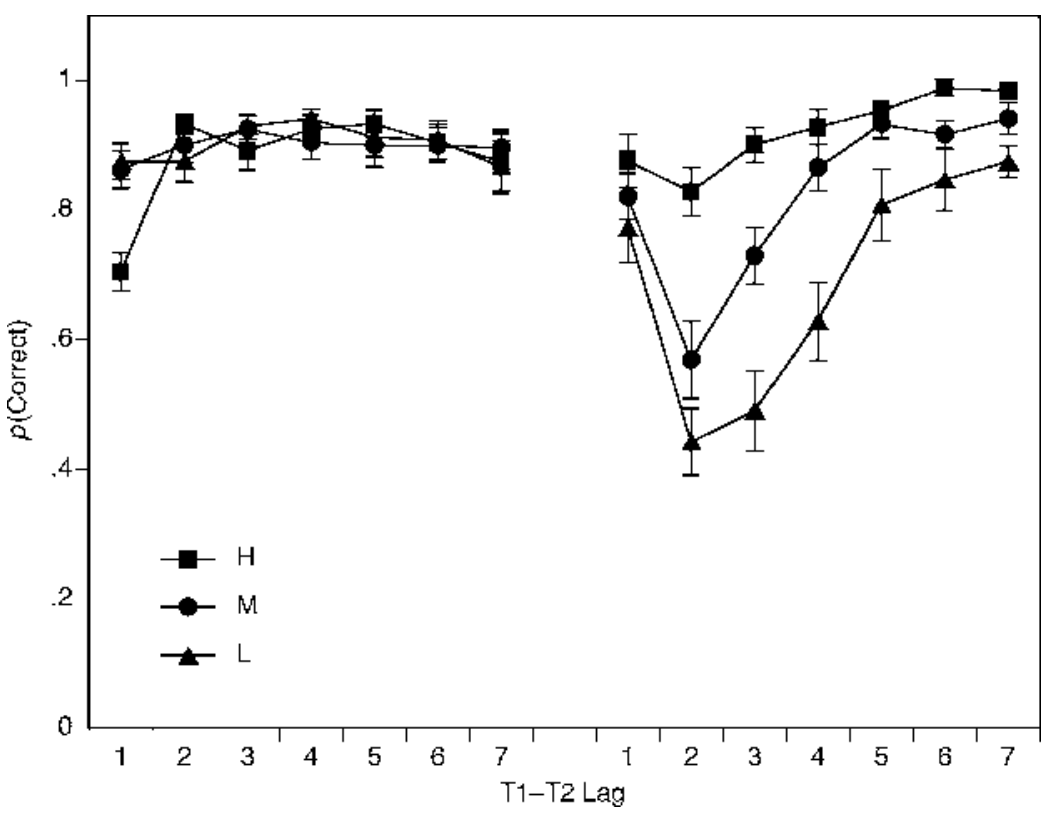

Figure 4. Experiment 3: identification performance as a function of T1-T2 lag. Left panel, $p(\mathrm{~T} 1)$; right panel, $p(\mathrm{~T} 2 \mid \mathrm{T} 1)$. The contrast of $\mathrm{T} 2$ was varied: square $=$ high, circle $=$ medium, and triangle $=$ low contrast. Error bars $=1 S E$.

more than compensated for by the protection obtained by increasing T2's contrast. For T1 performance, the difference between the high- and the low-contrast conditions was approximately $10 \%$ in both experiments. In Experiment 4A, T2 performance, at the height of the blink (lag 2), decreased by only $10 \%$ in the high-contrast condition but by more than $40 \%$ in the low-contrast condition. (T2 performance at lag 7 was used as the baseline, since the blink petered out here.) The comparable statistics for Experiment 4B were $38 \%$ and $51 \%$ for the highand the low-contrast conditions, respectively. The critical difference between the two experiments is that in Experiment $4 \mathrm{~A}$, the contrast was $75 \%$ for the high-contrast condition, but in Experiment 4B, it was only 50\%. The differences between the two experiments implied that T2's contrast - which protects against substitution masking (Experiment 3) - played the more critical role in modulating the effects of the blink.

Another way of looking at this issue is by comparing dwell times of Experiments 2A and 4A, which had the same T1 luminance profile. In Experiment 2A, the additional dwell time caused by a high-contrast $\mathrm{T} 1$ (as compared with the low-contrast T1) was 1.67 lags. In Experiment 4A, a high-contrast $\mathrm{T} 2$ reduced dwell time by more than 3.67 lags.

Relative target-distractor contrast and performance. So far, the analyses have focused on target contrast. But as the next set of analyses show, target contrast alone cannot explain the variation in performance. This point is best made by comparing the medium-luminance condition in Experiments 4A and 4B, which had identi- cal (40\%) target contrast. Performance was better in Experiment $4 \mathrm{~A}(\mathrm{AB}=62$, as compared with 155 for $\mathrm{Ex}-$ periment $4 \mathrm{~B})$. Results consistent with this trend were revealed in a 2 (experiment: 4A vs. 4B) $\times 7$ (lags) ANOVA on $\mathrm{T} 2$ performance. There was an overall experiment effect $\left[F(1,28)=13.408, M S_{\mathrm{e}}=0.172, p<.001\right]$, and the experiment $\times$ lag interaction failed to reach significance $\left[F(6,168)=2.018, M S_{\mathrm{e}}=0.023, p<.07\right]$. But although the absolute contrast was the same, these two conditions had different target:distractor (T/D) contrast ratios. In Experiment 4A, the T/D ratio was 3.2, and in Experiment $4 \mathrm{~B}$, it was 0.8 . As the T/D ratio increased, so did performance.

The results may be summarized as follows. First, as the contrasts of the targets increased, relative to the distractors, the magnitude of the $\mathrm{AB}$ diminished. In a sense, this must be trivially true. If the distractors' contrast is set so low that they are invisible, the $\mathrm{AB}$ should disappear altogether. Second, performance improved when the relative T/D contrast was large and declined as the ratio became smaller. We explain this pattern of results by appealing to the substitution-masking hypothesis (Di Lollo et al., 2000). A high-contrast T1 was recovered better but also produced a larger AB (Experiment 2). When there is some delay in attention engaging $\mathrm{T} 2$, its low-level codes cannot be immediately consolidated, making them vulnerable to replacement by the trailing distractor's codes (i.e., substitution masking). But if T2 can be protected from substitution masking by setting the contrast of the trailing distractor low, relative to the target, the effects of the blink become attenuated. 


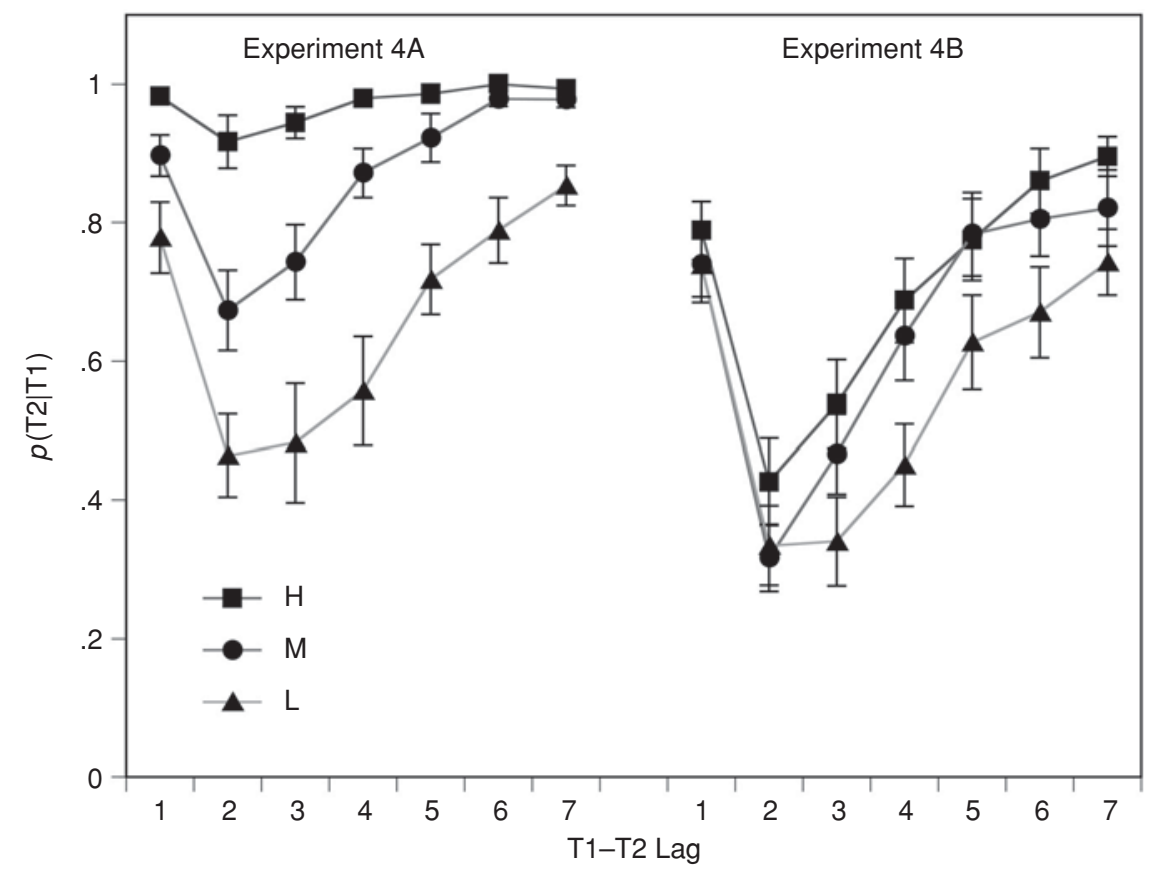

Figure 5. Experiments $4 A$ and $4 B$, left and right panels, respectively: probability of $T 2$ identification conditionalized on correct $\mathrm{T} 1$ as a function of $\mathrm{T} 1-\mathrm{T} 2$ lag. The contrasts of $\mathrm{T} 1$ and $\mathrm{T} 2$ were correlated: square $=$ high, circle $=$ medium, and triangle $=$ low contrast. Error bars $=1 S E$.

Di Lollo and his associates emphasized the importance of the visibility of the backward mask for substitution masking to succeed. The results of Experiment 4 showed that what is crucial was not so much the visibility of the mask per se as the energy of the mask, relative to the target. If attentional engagement of $\mathrm{T} 2$ is delayed, many more iterations of reentrant processing (Di Lollo et al., 2000) would be required for the low-level T2 codes to achieve a higher level representation. When the T/D ratio is low, the distractor's codes are more robust, as compared with T2's, thereby increasing the distractor's potential in replacing the target's codes. Conversely, when the T/D ratio is high, T2's codes, now more hardy, as compared with the codes of the trailing distractor, weather the delay in attentional engagement better, making substitution masking less likely. The overall pattern of results in Experiments 4A and 4B exemplify this point. The correlation between $\mathrm{T} / \mathrm{D}$ contrast and $\mathrm{AB}$ magnitude was -.972 .

\section{EXPERIMENT 5}

When a blank frame is inserted after T1, the blink disappears altogether, producing a flat lag function (Breitmeyer, Ehrenstein, Pritchard, Hiscock, \& Crisan, 1999; Grandison, Ghirardelli, \& Egeth, 1997; Raymond et al., 1992). Others (e.g., Chun \& Potter, 1995; Seiffert \& Di Lollo, 1997) have failed to replicate the flat lag function, although their results all showed that the blank improved performance. It is unclear why inserting a blank after $\mathrm{T} 1$ sometimes fails to eliminate $\mathrm{AB}$. In Experiment 2, a high-contrast $\mathrm{T} 1$ produced a larger $\mathrm{AB}$. The question is the following: Would introducing a blank have the same effect whether it was preceded by a highor a low-contrast T1?

\section{Method}

Procedure. The design of the experiment was a 2 (blank vs. baseline) $\times 2$ (contrast: high vs. low) $\times 5$ (lags: $1-5$ ) factorial. The observers identified two targets. When $\mathrm{T} 2$ was in the lag 1 position (i.e., lag $=1$ condition), the blank and the baseline conditions were identical: all the frames contained letters. For the other lags, the frame trailing $\mathrm{T} 1$ was empty in the blank condition, but in the baseline condition, all the frames were filled. The luminance of the high- and low-contrast $\mathrm{T} 1$ was 10 and $40 \mathrm{~cd} / \mathrm{m}^{2}\left(\right.$ contrast $\left.^{-}\right)$. The luminance of T2 $\left(60 \mathrm{~cd} / \mathrm{m}^{2}\right)$ was identical in all the trials. The background was $90 \mathrm{~cd} / \mathrm{m}^{2}$, and the distractors $120 \mathrm{~cd} / \mathrm{m}^{2}$. The SOA was $94 \mathrm{msec}$, with the letter frame displayed for $47 \mathrm{msec}$, followed by a blank of $47 \mathrm{msec}$.

The observers were 18 students recruited from the same pool. Each participated in 12 blocks, with the 1 st block regarded as practice and its data excluded from analysis.

\section{Results and Discussion}

The dependent variable was the accuracy of $\mathrm{T} 2$ identification conditioned on correct $\mathrm{T} 1$. The data are presented in Figure 6. Inserting a blank in the lag 1 position eliminated the $\mathrm{AB}$ when the $\mathrm{T} 1$ contrast was low (left plot), but not when the T1 contrast was high (right plot).

First, the data from the baseline trials were analyzed in a 2 (contrast) $\times 5$ (lags) ANOVA. A high-contrast T1 produced poorer overall $\mathrm{T} 2$ performance $[F(1,17)=$ 
34.920, $\left.M S_{\mathrm{e}}=0.014, p<.001\right]$, replicating the critical contrast effects of Experiment 2A. Performance varied across lags $\left[F(4,68)=8.118, M S_{\mathrm{e}}=0.045, p<.001\right]$. The contrast effect was modulated by the lag variable, with the T1 contrast effect diminishing as lag increases $\left[F(4,68)=6.963, M S_{\mathrm{e}}=0.064, p<.001\right]$.

Consider now the trials in which the blank was inserted. A 2 (contrast) $\times 4$ (lags 2-5) showed that a highcontrast $\mathrm{T} 1$ produced significantly worse performance $\left[F(1,17)=33.217, M S_{\mathrm{e}}=0.019, p<.001\right]$, again replicating Experiment $2 \mathrm{~A}$. Performance improved with lags $\left[F(3,51)=5.679, M S_{\mathrm{e}}=0.034, p<.005\right]$, and critically, the contrast effect was modulated by lags $[F(3,51)=$ $\left.5.230, M S_{\mathrm{e}}=0.015, p<.005\right]$. For the low-contrast $\mathrm{T} 1$ trials, a one-way ANOVA of the five lag (lags 2-6) conditions revealed a flat lag function $(F<1)$; the linear trend was also not significant $(F<1)$, replicating Raymond et al. (1992), Grandison et al. (1997), and Breitmeyer et al. (1999). In contrast, an analogous ANOVA for the high-contrast $\mathrm{T} 1$ showed a clear lag effect $[F(3,51)=$ $\left.8.713, M S_{\mathrm{e}}=0.028, p<.001\right]$ and a reliable linear trend $\left[F(1,17)=18.250, M S_{\mathrm{e}}=0.034, p<.001\right] .{ }^{9}$

Inserting the blank immediately after a high-contrast T1 improved performance even if it did not eliminate the AB, replicating Chun and Potter (1995) and Seiffert and Di Lollo (1997). A 2 (blank vs. baseline) $\times 4$ (lags 2-5) ANOVA for the high-contrast condition revealed that the blank improved performance $\left[F(1,17)=10.900, M S_{\mathrm{e}}=\right.$ $0.021, p<.001]$, as did the lag variable $[F(3,51)=$ $\left.13.342, M S_{\mathrm{e}}=0.034, p<.001\right]$. But critically, the two variables did not interact $(F<1)$. This result is impor- tant. If the effect of blank frame was merely facilitating T1 processing so that the attention shift could occur earlier, performance elevation should have obtained only when the lag was short and should have petered out when the lag was longer - that is, the interaction effect should have been reliable. There was no hint of an interaction effect: The blank elevated performance across all lags.

The pattern of results in Experiment 5 is consistent with that in Experiment 2. A higher contrast T1 produced a larger $\mathrm{AB}$. More important, whereas inserting a blank after a low-contrast $\mathrm{T} 1$ succeeded in eliminating the $\mathrm{AB}$, the same blank frame after a high-contrast $\mathrm{T} 1$ merely modulated the $\mathrm{AB}$ magnitude but failed to eliminate it.

\section{GENERAL DISCUSSION}

This set of five RSVP experiments examined how target contrast modulated the $\mathrm{AB}$. When attention selects a target and another appears shortly thereafter, attention needs to disengage from $\mathrm{T} 1$ before it can shift to and engage the new target (Posner \& Petersen, 1990). The contrast manipulation was aimed at influencing the early stages of code processing. The main findings can be summarized as follows.

1. A target with a higher contrast was identified more accurately (Experiments 1, 2, 4, and 5) and also was detected more quickly (Experiment 2B).

2. But increasing $\mathrm{T} 1$ 's contrast hampered $\mathrm{T} 2$ recovery. This relationship obtained whether attention was engaged exogenously (Experiment 1) or endogenously (Ex-

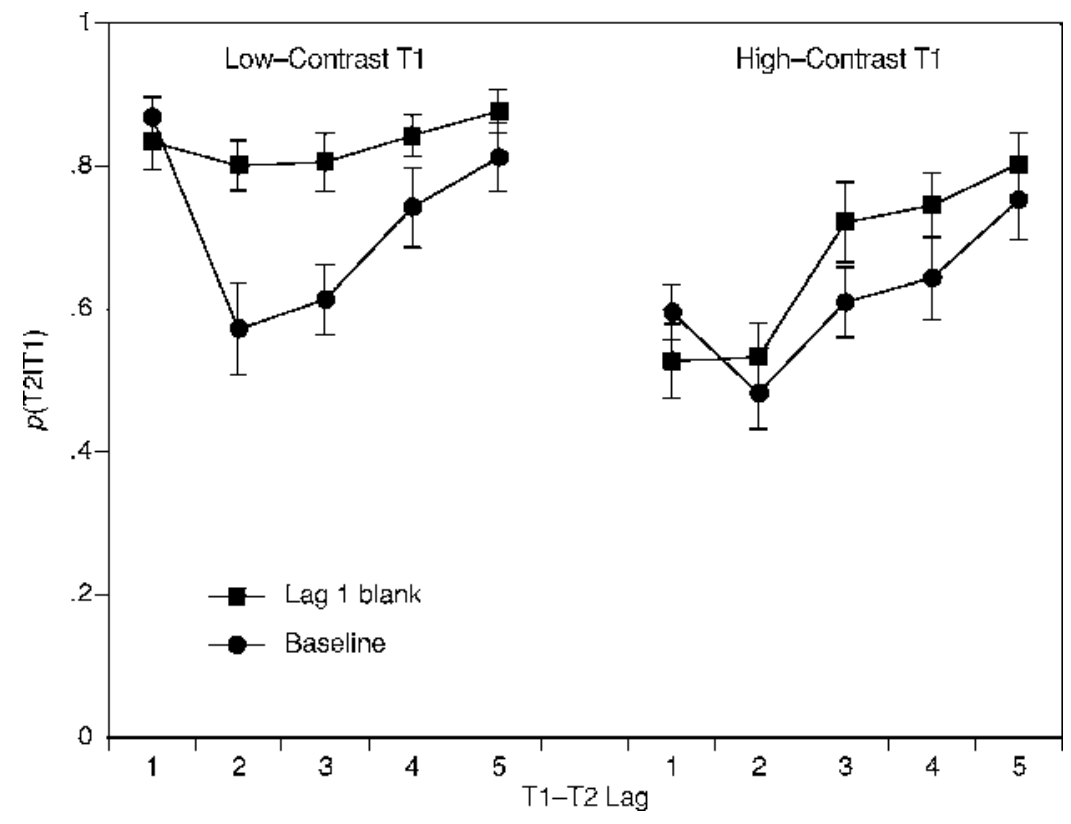

Figure 6. Experiment 5: probability of $\mathrm{T} 2$ identification conditioned on correct $\mathrm{T} 1$ as a function of T1-T2 lag. Left panel, T1 contrast low; right panel, T1 contrast high. Square = blank lag 1 frame; circle $=$ baseline. Error bars $=1 S E$. 
periment 2). Trailing T1 with a blank eliminated the AB only when T1's contrast was low. The blink persisted when T1's contrast was high (Experiment 5).

3. Performance improved when T2's contrast increased (Experiment 3). The higher T2 contrast also compensated for the longer dwell caused by a high-contrast T1 (Experiment 4).

\section{Target Contrast and Performance}

Stimulus contrast facilitates perceptual processing. The effects of luminance on visual ERPs are known to be manifest 70-80 msec after stimulus onset (Johannes, Münte, Heinze, \& Mangun, 1995). A high-contrast T1 led to faster detection response (Experiment 2B) and produced better identification performance (Experiments 2, 4, and 5). Pooled across the relevant experiments, ${ }^{10}$ the correlation $(r)$ between T1 performance and T1-distractor relative contrast was $.84(p<.001)$. These results either replicate or are consistent with those in the psychophysical literature (Ejima \& Otani, 1987; Gish et al., 1986; Lupp et al., 1976; Murray \& Plainis, 2003; Plainis \& Murray, 2000; Tiipana, Paramei, \& Alshuth, 2001).

\section{T1 Contrast and AB Models}

If target processing delays attentional disengagement, a high-contrast target, which facilitates an earlier onset of postperceptual consolidation, should allow an earlier attentional disengagement. According to the bottleneck models, the serial processing of a target (Chun \& Potter, 1995) or its postperceptual consolidation (Jolicœur, 1998) must be completed first before a new target (T2) may be engaged. Thus, any delay in T1 processing would have a knock-on effect on T2, delaying the latter's processing and, thus, its recovery. When processing of T1 is finally complete and attention eventually disengages, T2's codes may have, by this time, disintegrated so much that its identity is no longer retrievable. Thus, variables (such as the target's luminance) that facilitate perceptual processing should allow an earlier disengagement from the current target and thereby improve the recovery of the next target.

In regard to Raymond et al.'s (1992) shut-and-lock model, increasing T1's contrast should not provide more or less protection against binding errors. Thus, the model should predict that $\mathrm{T} 1$ contrast manipulation ought not to have any effect on the AB. The predictions of the interference model (Raymond et al., 1995; Shapiro, Raymond, \& Arnell, 1994) are less clear. It attributes the AB to the success of distractors in wresting away processing resources that would otherwise be allocated to the targets (specifically, T2). A template-matching procedure determines those distractors that would enter visual STM, and they would then contend with the targets for the limited resources. In their proposal, template matching is a feature-driven process. So long as the targets are suprathreshold, their contrast manipulations should not influence the outcome of this matching process and, thus, should have no effect on the AB. But if target-distractor luminance differences may be used to assign more resources to the targets, the prediction would be a smaller $\mathrm{AB}$ for a higher target contrast.

The results from these experiments show that the facilitation of $\mathrm{T} 1$ performance in fact produced worse $\mathrm{T} 2$ identification performance. Thus, although a high-contrast T1 was processed more quickly, paradoxically, attention seemed to have been detained longer at its site. For this set of experiments, the overall correlation between $\mathrm{T} 1$ performance and $\mathrm{AB}$ magnitude was not reliable $[r(14)=$ -.18 , n.s.].

\section{Exogenous Versus Endogenous Attention}

This seemingly paradoxical result - that enhancing T1's contrast facilitated its processing but hindered T2 recovery - is the central finding of this set of experiments. Faster processing and/or earlier engagement of $\mathrm{T} 1 \mathrm{did}$ not produce earlier disengagement and, thus, did not, in fact, allow an earlier engagement of $\mathrm{T} 2$.

The following account is based largely on the theoretical ideas developed by Loftus and his colleagues (Busey \& Loftus, 1994; Loftus, Duncan, \& Gehrig, 1992; Loftus \& Irwin, 1998; Loftus \& Ruthruff, 1994). Assume that the linear systems approach (Graham, 1989; Watson, 1986) provides a good first approximation to early visual processing. When the stimulus appears, the first stage of its processing involves low-pass temporal filtering, the result of which is a sensory response function describing (neural) activation over time. In the analysis of Loftus and his colleagues, what is critical is the area under the response function, which they claimed is proportional to the energy of the input stimulus and which they regarded as the total amount of information to be acquired (Loftus \& Ruthruff, 1994). Loftus and Ruthruff showed that performance was (at least) a monotonic function of energy. The strong correlation $(r=.84)$ between T1 contrast and performance found in these experiments is consistent with their view.

The sensory response function generated from a highcontrast stimulus covers a larger area and, critically, extends longer (see, e.g., the simulated functions in Busey \& Loftus, 1994). If information acquisition continues for the full extent of its availability (the area spanned by the function), performance would improve as contrast increases, but acquisition time would, correspondingly, lengthen. Busey and Loftus assumed that for any sensory response function, there was some threshold value below which information acquisition stopped. The high-contrast stimulus generates a larger function. The duration for which it stays above the threshold is longer. In Experiments 1,2 , and 5, we showed that a higher contrast stimulus is associated with a larger $\mathrm{AB}$, implying a longer dwell time.

The effect of stimulus contrast on AB magnitude was observed whether attention was exogenous (Experiment 1) 
or endogenous (Experiments 2 and 5). Recall that the cost of an exogenous $A B$ is smaller than that for the $A B$ produced when attention was endogenous. The literature distinguishes between a voluntary and an involuntary OR (e.g., Maltzman \& Langdon, 1982; Näätänen, 1992). This distinction parallels the one made between the transient and the sustained attentional mechanisms (Cheal \& Lyon, 1991; Nakayama \& Mackeben, 1989; Sperling \& Weichselgartner, 1995; Yantis et al., 2002) and, in the psychophysiological literature, between a call for attention and the allocation of attentional resources in answer to that call (Cowan, 1995; Näätänen, 1992; Öhman, 1979; Siddle, 1991). The overall sensory response function may be decomposed into two parts, one corresponding to the activation of transient, and the other to that of sustained mechanisms (e.g., Kulikowski \& Tolhurst, 1973).

In these experiments, the luminance manipulations meant that the target's appearance was always marked by a sudden change in the mean luminance of the display, which ought to produce an exogenous orienting to the stimulus. In the case of a higher contrast stimulus, the change is larger, and a larger transient component may be expected. Nakayama and Mackeben (1989) posited that the transient component is not subject to endogenous control and is time locked to the stimulus triggering the response. The sustained component, however, is under voluntary control; its temporal resolution is also longer.

In the discussion that follows, we will relate the different modes of attentional orienting to the overall shape of the sensory response function. According to Sokolov (1960), a neural representation (neuronal model) of a stimulus develops from repeated orienting toward it. This neuronal model encapsulates the specific neural activation from the stimulus. This idea is akin to the notion of the template in the models proposed by Chun and Potter (1995) and Shapiro et al. (1994) and also to Folk et al.'s (1992) conception of the ACS. As the letters stream in, each is compared with the template and, upon a match, the item enters a temporary processing buffer. The twostage and the interference models assume only a single target-defined template. Matching to this template filters out all but the targets. Yet the results of Experiment 1 showed that the singleton captured attention despite the fact that neither its luminance profile nor its features fit this template. The question is how a nonrelevant stimulus (five spots) could succeed in triggering an OR (and produce an $\mathrm{AB})$ ?

Gati and Ben-Shakhar (1990) argued that since both novelty and significance are known to produce orienting to a stimulus, there must be some underlying mechanism to distinguish between exogenous and endogenous orienting. They posited two separate neuronal models: one tuned to the standard stimulus (e.g., the distractors in these experiments) and the other tuned to the task-relevant (target) dimensions. Matching to the standard is much like sensory filtering described, say, in the original Broad- bent model (Cowan, 1995). Accordingly, the distractors would be filtered out as they matched the standard model (a specific luminance plus letter features). But when there is a mismatch, an involuntary OR (an orienting reflex), corresponding to the transient component of the sensory response function, is evoked. The mismatch triggers a further check to ascertain whether the stimulus causing the mismatch is, in fact, a target. The check is obtained by matching the stimulus to the task-relevant neuronal model. When there is a match, endogenous orienting ensues, and the sustained component of the sensory function is evoked at this juncture.

The singleton (Experiment 1) produced a mismatch when compared with the standard model, and it triggered an involuntary OR. But it would fail to match the taskrelevant model, and thus, no voluntary OR was evoked. The amplitude of its sensory response function, consisting only of the transient component, was determined by the magnitude of the mismatch. When the difference between target and distractor luminance increased, the mismatch was correspondingly larger, producing a sensory function of greater amplitude. Dwell time was thus longer, as was shown in Experiment 1. This had two consequences. First, when a larger OR was evoked, targets that appeared some lags away continue to enjoy the sparing effect. Second, when the OR was larger, there was a greater likelihood of intrusions by distractors trailing the singleton. Information acquisition appeared to begin just as soon as attention was engaged, whether the engagement was due to exogenous or endogenous orienting.

Capture by a singleton can sometimes be evaded, as Raymond et al. (1992, Experiment 2) showed. In their experiment, the singleton (a white letter) was shown on every trial. It may be expected that, over time, the singleton's appearance would have been incorporated into the neuronal model. When observers expect to see a white letter, the neuronal model of the standard stimulus will be configured to include this singleton's features. Not surprisingly, then, when the white letter appears, there is now a match to the standard model. Consequently, no exogenous OR was evoked, and no capture was observed.

In this analysis, attention is assumed to disengage only when the overall sensory response function goes below threshold. In a two-target RSVP task, one possible strategy is for the visual system to acquire just enough information (some minimum area under the sensory response function) for the identification response to be computed, at which point information acquisition stops and attention disengages. For a high-contrast target, less time is needed to acquire this minimum amount, making possible an earlier attentional disengagement. The net result should be a smaller AB. Conceptually, the system acts as if speed were crucial for the T1 task. Indeed, when speed was emphasized (Experiment 2B), this was probably the strategy. But when speed was not consequential (Experiment 2A), information continued to be acquired as long as it was available. 


\section{Attentional Blink}

Recent evidence (Folk et al., 2002; Wee \& Chua, 2004) has shown that attention can be captured even when the system is undergoing an $\mathrm{AB}$. But capturing attention, when the blink is already at large, implies that attention has to engage at the capture (temporal) site, although the overall sensory response function is still above the threshold (e.g., when the capture stimulus appears soon after the target). But recall that the exogenous and the endogenous attentional systems are separate and independent (e.g., LaBerge, 2002). The transient component has a shorter temporal resolution; the sustained component arrives later and lasts longer. When a new target or a novel object pops out, the involuntary OR to the previous object may already have subsided, making possible the triggering of a new involuntary OR to the new target. But the corresponding sustained component to the previous object may not have petered out, and no sustained component can be triggered immediately for the new target. This severely limits the amount of information that may be acquired about the new object. Thus, observers sense that $\mathrm{T} 2$ has appeared but will fail to identify it. When two targets appear in close temporal proximity, observers sometimes register only one target. Here, the involuntary OR for T2 cannot be triggered because the OR to T1 has not subsided. This argument is developed further by considering two phenomena: (1) lag 1 sparing and (2) the elimination of the $\mathrm{AB}$ when a blank is inserted after $\mathrm{T} 1$.

Lag 1 sparing. When two targets appear consecutively, T2 is identified better than when a distractor intervened between them. This lag 1 sparing phenomenon (Potter et al., 1998) may be explained in terms of the overlap between the targets' sensory response functions. The overlap of the functions depends on, among other things, the interstimulus interval (ISI) and the similarity between the stimuli. The effects of these two variables may be complementary and are considered in turn. Consider, first, the ISI. If the two stimuli were contemporaneous, only a single function would be generated, reflecting the sum of their energies. As the ISI increases, the overlap decreases accordingly. The ISI in these experiments, less than $50 \mathrm{msec}$, was probably short enough for the response functions generated by the target and the trailing stimulus to overlap somewhat. Therefore, the function from which information is acquired would (potentially) be a composite of these two overlapping functions. This composite function falls below threshold later. Conceptually, this is analogous to the notion of an attentional system with a sluggish gating device, wherein both the target and the trailing distractor(s) get admitted into the processing buffer (Chun \& Potter, 1995; Raymond et al., 1992; Visser, Bischof, \& Di Lollo, 1999). Seiffert and Di Lollo (1997) advanced a similar temporal integration argument.

Next, consider the role of target-distractor similarity. There have been several demonstrations of the relationship between target-distractor similarity and the $\mathrm{AB}$ (Chun \& Potter, 1995; Raymond et al., 1995; Shapiro et al., 1994). Chun and Potter (1995, Experiments 4 and 5) also showed that local similarity was more important than global similarity. According to Dixon and Di Lollo's (1994) temporal-coding hypothesis, if the stimulation provided by temporally contiguous time slices correlated highly, they would be interpreted as deriving from the same event. When the temporally adjacent stimuli are similar (e.g., same luminance or similar features), there would be a larger correlation between their response functions, and this increases the likelihood that the two events will be coded as coterminous. Information would then be acquired from a larger composite function, thereby prolonging the acquisition phase. When the temporally adjacent stimuli are targets, lag 1 sparing occurs, because information about both may now be acquired from a single function. Performance improves because attention need not disengage first from one target and then reengage at another, as would be necessary when one or more distractors intervened between the targets. When the functions the targets generate do not form a composite (e.g., targets in different locations), their recovery from a single function would not be possible, and thus lag 1 sparing would not be observed (Breitmeyer et al., 1999). Consistent with this analysis, we observed that the sparing effect declined as T1's contrast increased (Experiments 2A and 3; see Table 1). In Experiment 4B, in which the contrasts of T1 and T2 were correlated, there was a smaller discrepancy between the different contrasts. (The results of Experiment $4 \mathrm{~A}$ are less consistent, probably because ceiling effects, especially in the high-contrast condition, were obtained.)

But recovering both targets from a single function comes at a cost: Their temporal order is sometimes reversed. The reversal pattern (see Table 1) observed in these experiments provides further insight into this phenomenon. When the targets' contrasts are more similar, reversals are more likely. In Experiments 2A and 3, when $\mathrm{T} 1$ and $\mathrm{T} 2$ had identical (low) contrast, the reversal rate was .12-.14. But when the target contrasts were different, the highest reversal rate was only .06. When temporally adjacent stimuli have the same contrasts, their separate sensory response function is more likely to be treated as coextensive. The two targets are recovered from a single function, and as a result, reversal rates increase.

Blank after T1. Inserting a blank after T1 eliminates the AB (Raymond et al., 1992). The blank increases the ISI between the T1 and the next distractor, thus decreasing the likelihood that their response functions will overlap. With no overlap, information can be acquired only from the T1 function. This function falls below the threshold earlier, allowing attentional disengagement to occur sooner, the net result of which is a smaller (or negligible) $\mathrm{AB}$. In Experiment 5, the $\mathrm{AB}$ was, by and large, eliminated when a blank was inserted after a low-, but not after a high-, contrast T1. In the high-contrast condition, the sensory function generated was large enough to bridge the blank gap on some large proportion of trials. Thus, it was more likely that there would be an overlap with the 
function generated by the lag 2 distractor. Now, information was again acquired from a composite function. Seiffert and Di Lollo (1997, Experiment 2) showed that when T1 was simultaneously masked and a blank followed the $\mathrm{T} 1+$ mask frame, the AB was not eliminated. Simultaneous masking probably produced an energy profile of the target frame that was roughly the sum of both the target and the mask luminance. Thus, the net effect was probably not unlike the high-contrast condition in Experiment 5.

\section{T2 Contrast and AB}

A high-contrast T1 produces a larger AB (Experiment 2) because attention engages at $\mathrm{T} 1$ for as long as information is available to be acquired. There is a longer dwell time, which enhances T1 identification. But there will be a bigger delay in $\mathrm{T} 2$ processing. By the time attention engages at $\mathrm{T} 2$, its codes can have deteriorated substantially. More iterations of reentrant processing will be required for these codes to achieve a higher level representation on which identification relies (Di Lollo et al., 2000). Under this scenario, a stronger representation is obtained from the codes of the distractor trailing T2, making the distractor more likely to succeed as a mask of the preceding target. But as the results of Experiment 4 showed, T2's contrast (relative to the distractors) offers some measure of protection against substitution masking. As its contrast increases, the response function generated for $\mathrm{T} 2$ is larger and extends longer. The net result is that the target's codes survive the delay in attentional engagement better.

The critical variable is not (just) the contrast of $\mathrm{T} 2$ or the visibility of the distractor trailing $\mathrm{T} 2$ but their relative T/D contrast. This point is best seen by comparing the $\mathrm{AB}$ magnitudes in the medium-contrast conditions in Experiments $4 \mathrm{~A}$ and $4 \mathrm{~B}$, which are identical (40\% contrast). The AB magnitudes were 62 and 155, for Experiments $4 \mathrm{~A}$ and $4 \mathrm{~B}$, respectively. As the T/D ratio increased, $\mathrm{AB}$ decreased in magnitude (see Table 1). If T2's contrast is low relative to the trailing distractor, the latter's codes stand a better chance of reaching consciousness first and, thereby, succeed in masking T2. Conversely, if T2's contrast is high relative to the distractor, the target's codes are more likely to be transformed to its higher representation first. In the limit, if the distractor is invisible $(\mathrm{T} / \mathrm{D}=\infty)$, the trailing distractor stands no chance at all in masking $\mathrm{T} 1$. This was the boundary condition investigated by Giesbrecht and Di Lollo (1998): The AB was eliminated when the RSVP sequence ended on T2.

\section{Conclusion}

This account accommodates several key insights from the extant AB literature: (1) Masking T1 exacerbates the $\mathrm{AB},(2)$ similarity between a target and a distractor also exacerbates the AB, (3) local similarity between a target and a distractor is more critical than global similarity, and (4) performance is better when the two targets appear consecutively. In this account, it is argued that what determines attention dwell time and, thus, AB magnitude is the amount of information available to be acquired. The presentation parameters of the typical RSVP experiment (ISI $<50 \mathrm{msec}$ ) almost guarantee that when attention engages, the response function is likely to be a composite of T1 and the trailing distractor. When the target and the distractors are sampled from the same set, local similarity is enhanced, and this increases the probability that the target and the trailing distractor are coded as arising from the same event. Two targets appearing consecutively are even more likely to be coded as coterminous. This improves their identification, and as a consequence, lag 1 sparing is observed. But it comes at a cost. When T1 and T2 are coded as coterminous, their temporal order is sometimes harder to recover.

\section{REFERENCES}

Albrecht, D. G., \& Hamilton, D. B. (1982). Striate cortex of monkey and cat: Contrast response function. Journal of Neurophysiology, $\mathbf{4 8}$ 217-237.

Breitmeyer, B. G. (1984). Visual masking: An integrative approach. Oxford: Oxford University Press.

Breitmeyer, B. G., Ehrenstein, A., Pritchard, K., Hiscock, M., \& CRISAN, J. (1999). The roles of location specificity and masking mechanisms in the attentional blink. Perception \& Psychophysics, 61, 798-809.

Broadbent, D. E., \& Broadbent, M. H. P. (1987). From detection to identification: Response to multiple targets in rapid serial visual presentation. Perception \& Psychophysics, 42, 105-113.

Busey, T. A., \& Loftus, G. R. (1994). Sensory and cognitive components of visual information acquisition. Psychological Review, 101, 446-469.

Cheal, M., \& Lyon, D. R. (1991). Central and peripheral precuing of forced-choice discrimination. Quarterly Journal of Experimental Psychology, 43A, 859-880.

Chun, M. M., \& Potter, M. C. (1995). A two-stage model for multiple target detection in rapid serial visual presentation. Journal of Experimental Psychology: Human Perception \& Performance, 21, 109127.

Cowan, N. (1995). Attention and memory: An integrated framework. New York: Oxford University Press.

Daffner, K. R., Mesulam, M. M., Scinto, L. F. M., Cohen, L. G., Kennedy, B. P., West, W. C., \& Holcomb, P. J. (1998). Regulation of attention to novel stimuli by frontal lobes: An event-related potential study. NeuroReport, 9, 787-791.

Di Lollo, V., Enns, J. T., \& Rensink, R. A. (2000). Competition for consciousness among visual events: The psychophysics of reentrant visual processes. Journal of Experimental Psychology: General, 129, 481-507.

Dixon, P., \& Di Lollo, V. (1994). Beyond visible persistence: An alternative account of temporal integration and segregation in visual processing. Cognitive Psychology, 26, 33-63.

DonNer, K. (1989). Visual latency and brightness: An interpretation based on the responses of rods and ganglion cells in frog retina. $\mathrm{Vi}$ sual Neuroscience, 3, 39-51.

Duncan, J., Ward, R., \& ShapIRo, K. L. (1994). Direct measurement of attention dwell time in human vision. Nature, 369, 313-315.

Ejima, Y., \& Ohtani, Y. (1987). Simple reaction time to sinusoidal grating and perceptual integration time: Contributions of perceptual and response processes. Vision Research, 27, 269-276.

Folk, C. L., Leber, A. B., \& Egeth, H. E. (2002). Made you blink! Contingent attentional capture produces a spatial blink. Perception \& Psychophysics, 64, 741-753.

FolK, C. L., \& Remington, R. W. (1998). Selectivity in distraction by irrelevant featural singletons: Evidence for two forms of attentional capture. Journal of Experimental Psychology: Human Perception \& Performance, 24, 847-858. 
Folk, C. L., Remington, R. W., \& Johnston, J. C. (1992). Involuntary covert orienting is contingent on attentional control settings. Journal of Experimental Psychology: Human Perception \& Performance, 18, 1030-1044.

Folk, C. L., Remington, R. W., \& Johnston, J. C. (1993). Contingent attention capture: A reply to Yantis (1993). Journal of Experimental Psychology: Human Perception \& Performance, 19, 682-685.

Gati, I., \& Ben-Shakhar, G. (1990). Novelty and significance in orientation and habituation: A feature-matching approach. Journal of Experimental Psychology: General, 119, 251-263.

Giesbrecht, B., \& Di LoLLo, V. (1998). Beyond the attentional blink: Visual masking by object substitution. Journal of Experimental Psychology: Human Perception \& Performance, 24, 1454-1466.

Gish, K., Shulman, G. L., Sheehy, J. B., \& Leibowitz, H. W. (1986). Reaction times to different spatial frequencies as a function of detectability. Vision Research, 26, 745-747.

Graham, N. (1989). Visual pattern analyzers. New York: Oxford University Press.

Grandison, T. D., Ghirardelli, T. G., \& Egeth, H. E. (1997). Beyond similarity: Masking of the target is sufficient to cause the attentional blink. Perception \& Psychophysics, 59, 266-274.

Johannes, S., Münte, T. F., Heinze, H. J., \& Mangun, G. R. (1995). Luminance and spatial attention effects on early visual processing. Cognitive Brian Research, 2, 189-205.

JoLICEUR, P. (1998). Modulation of the attentional blink by on-line response selection: Evidence from speeded and unspeeded Task ${ }_{1}$ decisions. Memory \& Cognition, 26, 1014-1032.

JOLICEUR, P. (1999). Concurrent response-selection demands modulate the attentional blink. Journal of Experimental Psychology: Human Perception \& Performance, 25, 1097-1113.

Jolicceur, P., \& Dell'ACQUa, R. (2000). Selective influence of second target exposure duration and Task $_{1}$ load effects in the attentional blink phenomenon. Psychonomic Bulletin \& Review, 7, 472-479.

KASTNER, S., \& UNGERLEIDER, L. G. (2000). Mechanisms of visual attention in the human cortex. Annual Review of Neuroscience, 23, 315-341.

Kulikowski, J. J., \& Tolhurst D. J. (1973). Psychophysical evidence for sustained and transient detectors in human vision. Journal of Physiology, 232, 149-162.

LaBerge, D. (2002). Attentional control: Brief and prolonged. Psychological Research, 66, 220-233.

Loftus, G. R., Duncan, J., \& Gehrig, P. (1992). On the time course of perceptual information that results from a brief visual presentation. Journal of Experimental Psychology: Human Perception \& Performance, 18, 530-549.

Loftus, G. R., \& IRwIN, D. E. (1998). On the relations among different measures of visible and informational persistence. Cognitive Psychology, 35, 135-199.

LoFTUS, G. R., \& RUTHRUFF, E. R. (1994). A theory of visual information acquisition and visual memory with special application to intensityduration tradeoffs. Journal of Experimental Psychology: Human Perception \& Performance, 20, 33-50.

Lupp, U., Hauske, G., \& Wolf, W. (1976). Perceptual latencies to sinusoidal gratings. Vision Research, 16, 969-972.

Maltzman, I., \& LANGDON, B. (1982). Novelty and significance as determiners of the GSR index of the orienting reflex. Physiological Psychology, 10, 229-234.

McLaughlin, E. B., Shore, D. I., \& Klein, R. M. (2001). The attentional blink is immune to masking-induced data limits. Quarterly Journal of Experimental Psychology, 54A, 169-196.

MurRaY, I. J., \& Plainis, S. (2003). Contrast coding and magno/parvo segregation revealed in reaction time studies. Vision Research, $\mathbf{4 3}$, 2707-2719.

Musselwhite, M. J., \& JefFreys, D. A. (1985). The influence of spatial frequency on the reaction times and evoked potentials recorded to grating pattern stimuli. Vision Research, 25, 1545-1555.

NäÄTÄNEN, R. (1992). Attention and brain function. Hillsdale, NJ: Erlbaum.

Nakayama, K., \& Mackeben, M. (1989). Sustained and transient components of focal visual attention. Vision Research, 29, 1631-1646.
Öhman, A. (1979). The orienting response, attention, and learning: An information-processing perspective. In H. D. Kimmel, E. H. van Olst, \& J. F. Orlebeke (Eds.), The orienting reflex in humans (pp. 443-471). Hillsdale, NJ: Erlbaum.

Pelli, D. G., \& Zhang, L. (1991). Accurate control of contrast on microcomputer displays. Vision Research, 31, 1337-1350.

Plainis, S., \& MurRaY I. J. (2000). Neurophysiological interpretation of human visual reaction times: Effect of contrast, spatial frequency and luminance. Neuropsychologia, 38, 1555-1564.

Posner, M. I. (1980). Orienting of attention. Quarterly Journal of Experimental Psychology, 32, 3-25.

Posner, M. I., \& Petersen, S. E. (1990). The attention system of the human brain. Annual Review of Neuroscience, 13, 25-42.

Potter, M. C., Chun, M. M., Banks, B. S., \& Muckenhoupt, M. (1998). Two attentional deficits in serial target search: The visual attentional blink and an amodal task-switch deficit. Journal of Experimental Psychology: Human Perception \& Performance, 24, 979-992.

Raymond, J. E., Shapiro, K. L., \& Arnell, K. M. (1992). Temporary suppression of visual processing in an RSVP task: An attentional blink? Journal of Experimental Psychology: Human Perception \& Performance, 18, 849-860.

Raymond, J. E., Shapiro, K. L., \& Arnell, K. M. (1995). Similarity determines the attentional blink. Journal of Experimental Psychology: Human Perception \& Performance, 21, 653-662.

ReICH, D. S., Mechler, F., \& Victor, J. D. (2001). Temporal coding of contrast in primary visual cortex: When, what, and why. Journal of Neurophysiology, 85, 1039-1050.

Seiffert, A. E., \& Di Lollo, V. (1997). Low-level masking in the attentional blink. Journal of Experimental Psychology: Human Perception \& Performance, 23, 1061-1073.

Shapiro, K. L., RaYmond, J. E., \& Arnell, K. M. (1994). Attention to visual pattern information produces the attentional blink in rapid serial visual presentation. Journal of Experimental Psychology: Human Perception \& Performance, 20, 357-371.

Siddle, D. A. T. (1991). Orienting, habituation, and resource allocation: An associative analysis. Psychophysiology, 28, 245-249.

SoKolov, E. N. (1960). Neuronal models and the orienting reflex. In M. A. B. Brazier (Ed.), The central nervous system and behavior (pp. 187-276). Madison, NJ: Madison Printing.

Sperling, G., \& Weichselgartner, E. (1995). Episodic theory of the dynamics of spatial attention. Psychological Review, 102, 503-532.

ThOMPSON, D., \& DrasDo, N. (1989). The effect of stimulus contrast on the latency and amplitude of the pattern electroretinogram. Vision Research, 29, 309-313.

Tiipana, K., Paramei, G. V., \& Alshuth, E. (2001). Contrast discrimination and choice reaction times at near-threshold pedestals. Spatial Vision, 15, 25-43.

Tolmurst, D. J., \& Movshon, A. J. (1975). Spatial and temporal contrast sensitivity of striate cortical neurons. Nature, 257, 674-675.

Turpin, G., Schaefer, F., \& Boucsein, W. (1999). Effects of stimulus intensity, risetime, and duration on autonomic and behavioral responding: Implications for the differentiation of orienting, startle, and defense responses. Psychophysiology, 36, 453-463.

Visser, T. A. W., Bischof, W. F., \& Di Lollo, V. (1999). Attentional switching in spatial and non-spatial domains: Evidence from the attentional blink. Psychological Bulletin, 125, 458-469.

Ward, R., Duncan, J., \& SHaPIRo, K. (1996). The slow time-course of visual attention. Cognitive Psychology, 30, 79-109.

Watson, A. B. (1986). Temporal sensitivity. In K. R. Boff, L. Kaufman, \& J. P. Thomas (Eds.), Handbook of perception and human performance: Vol 1. Sensory processes and perception (pp. 6-1 to 6-43). New York: Wiley.

Wee, S., \& ChUA, F. K. (2004) Capturing attention when attention "blinks." Journal of Experimental Psychology: Human Perception \& Performance, 30, 598-612.

Yantis, S. Schwarzbach, J., Serençes, J. T., Carlson, R., Steinmetz, M. A., Pekar, J. J., \& Courtney, S. M. (2002). Transient neural activity in human parietal cortex during spatial attention shifts. Nature Neuroscience, 5, 995-1002. 


\section{NOTES}

1. Raymond, Shapiro, and their associates (e.g., Raymond, Shapiro, \& Arnell, 1995; Shapiro, Raymond, \& Arnell, 1994) have proposed an alternate (interference) model. Some of its assumptions are similar to the two-stage model, but it proposes a different explanation for the $\mathrm{AB}$. The model assumes that all the processing occurs off line (when the RSVP sequence halts). Since the model assumptions do not fit the engage-shift-disengagement framework of Posner and Petersen (1990), it will not be described here. In the General Discussion section, the implications of the results for the interference model will be considered.

2 . The experiments of Jolicœur and his associates directly measure the duration of the postperceptual (decision) stage, and not the perceptual processing of $\mathrm{T} 1$ as such.

3. The luminance measurements, obtained using a Minolta LS-110 photometer from a distance of approximately $60 \mathrm{~cm}$, were made from a circular disk drawn on the monitor. The particular "gray" RGB value corresponding to the luminance value was used to draw the letters.

4. Contrast $=$ (stimulus luminance - background luminance $) /$ (stimulus luminance + background luminance). Thus, if the singleton is $10 \mathrm{~cd} / \mathrm{m}^{2}$ and the background is $70 \mathrm{~cd} / \mathrm{m}^{2}$, its contrast $=(10-70) /$ $(10+70)=-0.75$ (i.e., contrast $\left.{ }^{-}\right)$.

5. Attention dwell time (in lag units) is computed by comparing T2 performance at each lag with the baseline, using the algorithm described in McLaughlin et al. (2001). Briefly, if T2 performance is lower than the baseline by some criterion, the dwell time estimate is increased by 1 unit. McLaughlin et al. used the arbitrary criterion of $15 \%$. T1 performance was taken to be the baseline. [The assumption was that when the blink was over, $\mathrm{T} 1$ and $\mathrm{T} 2$ performance would be independent-i.e., $p(\mathrm{~T} 2)=p(\mathrm{~T} 1)$.] The magnitude of the $\mathrm{AB}$ is computed by summing the difference between baseline and $\mathrm{T} 2$ performance across those lags where the blink is in force. This formula is different from the one proposed by Shapiro, Raymond, and Arnell (1994), which summed the deviation of T2 performance from the baseline (which they set at 1.0) across all lags.

6 . The baseline was computed from trials in which the spots were absent (cf. McLaughlin et al.'s [2001] algorithm, which used T1 performance as the baseline).

7. The estimates were calculated for lags 1-7, using T1 performance as the baseline, as per McLaughlin et al. (2001). For the baseline estimate, lag 1 was excluded because a high-contrast T2 appeared to "mask" a lower contrast T1. (This masking disappeared after lag 1. See, e.g., the left panels of Figures 4 and 5.) Including lag 1 would mean that the baselines would be lower for the high-contrast conditions, thus elevating the estimates of dwell time and the $\mathrm{AB}$.

8. The 0.5 lag difference between the two experiments was not reliable. The difference in $\mathrm{AB}$ magnitude, however, was $[F(1,39)=5.207$, $\left.M S_{\mathrm{e}}=5732, p<.05\right]$. But the point that the OR component cannot be the sole determinant of dwell time still holds.

9. Analyses of the dwell time and $\mathrm{AB}$ magnitude data did not provide clear convergence. The blank modulated the effects. For AB magnitude, the critical contrast $\times$ blank/no-blank interaction effect was only marginally reliable $(p<.06)$. The interaction was not significant for the ANOVA on dwell time.

10. The correlation was computed with 14 points ( 3 each for Experiments 2A, 3, 4A, and 4B and 2 for Experiment 5 [no blank trials]). Average T1 performance was estimated from lags 2-5 for Experiment 5 and from lags $2-7$ for the rest.

(Manuscript received June 3, 2003; revision accepted for publication October 4, 2004.) 